\title{
El financiamiento de los proyectos de carbono forestal: Experiencias existentes y oportunidades en México
}

\section{The financing of forest carbon projects: Existing experiences and opportuni- ties in Mexico}

\author{
Alejandro Ranero ${ }^{*}$ y Sara Covaleda'
}

1 Kibeltik Clima y Medio Ambiente A.C. San Cristóbal de * Autor de correspondencia. alejandro.ranero@gmail.com las Casas, Chiapas, México.

\begin{abstract}
RESUMEN
Este trabajo presenta una síntesis de los mecanismos de financiamiento empleados para la mitigación de emisiones en el sector forestal a escala internacional y las experiencias desarrolladas en México. A su vez, se analizan las oportunidades de mitigación del sector Uso del Suelo, Cambio de Uso del Suelo y Silvicultura [Uscusys] mexicano para cumplir con los objetivos nacionales. A escala internacional, los proyectos forestales de carbono han participado en mercados voluntarios y de cumplimiento, aunque los proyectos de países en vías de desarrollo han tenido un papel limitado en el marco del Protocolo de Kioto. El desarrollo de nuevos mecanismos de mitigación en la Comisión Marco de las Naciones Unidas sobre el Cambio Climático ha supuesto la necesidad de ampliar los enfoques para su financiamiento, que se ha materializado en acuerdos bilaterales y multilaterales en el caso del mecanismo de Reducción de Emisiones por Deforestación y Degradación forestal más la conservación, el manejo forestal sustentable y el incremento de los almacenes forestales [REDD+], en distintos países. En México, la participación del sector forestal en los mercados voluntarios y mecanismo para un desarrollo limpio ha sido, hasta el momento, limitada. Sin embargo, el país se ha preparado para la implementación de un mecanismo REDD+ a escala nacional y ha puesto en marcha su Iniciativa de Reducción de Emisiones [IRE]. Para alcanzar los objetivos de reducción de emisiones del sector Uscusys, México debe lograr una efectiva coordinación entre políticas forestales y de cambio climático y un óptimo aprovechamiento de los mecanismos financieros que se están gestando, destacándose la experiencia de la IRE y el desarrollo de un esquema regulado de comercio de emisiones a escala nacional. En este sentido, la aceptación de créditos de compensación de distintos tipos de proyectos forestales, en una visión más amplia a la contemplada en el pago opcional del impuesto al carbono a través de bonos de carbono, podría traducirse en importantes beneficios medioambientales, económicos y sociales.
\end{abstract}

Palabras Clave: impuesto al carbono, mercados de carbono, nuevos mecanismos de mitigación, REDD+.

\section{ABSTRACT}

This paper presents a synthesis of the financing mechanisms used internationally for the mitigation of greenhouse gas emissions in the forestry sector, and the experiences developed in Mexico. In addition, the mitigation opportunities of the Land Use, Land Use Change and Forestry sector [Lulucf] to meet national goals are analyzed. At the international level, forest carbon projects have participated in voluntary and compliance market schemes although forest projects from developing countries have had a limited role under the Kyoto Protocol. The development of new mitigation mechanisms under the United Nations Framework Convention on Climate Change [Unfccc] implied the need to expand the financing approaches, and the bilateral and multilateral agreements for the Reduction of Emissions from Deforestation and Forest Degradation plus conservation, increase of carbon stocks and sustainable forest management [REDD+] mechanism in different countries, are examples of that. In Mexico, the participation of the forestry sector in the voluntary carbon market and the Clean Development Mechanism has been limited. However, the country engaged in the preparation of a REDD+ mechanism at the national level, and is implementing its Emissions Reduction Initiative [ERI]. To achieve emissions reduction goals in the Lulucf sector, Mexico must achieve an effective coordination between forestry and climate change policies and an optimal use of the financial mechanisms that are being developed, specially the ERI's experience and the development of an Emission Trading Scheme at the national level. In this regard, the acceptance of offset credits from different types of forestry projects, in a broader perspective than that contemplated for the optional payment of the carbon tax through carbon credits, could result in important environmental, economic and social benefits.

KEYWORDs: carbon tax, carbon markets, new mitigation mechanisms, REDD+. 


\section{INTRODUCCIÓN}

A escala mundial, las emisiones procedentes del sector Agricultura, Forestería y Otros Usos del Suelo [Afous] se han estabilizado desde el año 2000, aunque su contribución a las emisiones globales sigue siendo elevada ( $24 \%$ en 2010; Intergovernmental Panel on Climate Change [IPCC], 2014). Se han observado importantes variaciones entre los países desarrollados -en los cuales las emisiones del sector Afous provienen principalmente de las actividades agrícolas- y en los países en vías de desarrollo, en los que las emisiones proceden, en mayor medida, de la deforestación y degradación forestal (IPCC, 2014).

En México, el Inventario Nacional de Gases de Efecto Invernadero [Inegei], actualizado para el año 2013, indica que las emisiones del sector Agricultura constituyeron $12 \%$ (80 169 Gg) de las emisiones totales y las emisiones asociadas al sector Uso del Suelo, Cambio de Uso del Suelo y Silvicultura (Uscusys) constituyeron 4.9\% (32 424 Gg) (Instituto Nacional de Ecología y Cambio Climático [Inecc]-Secretaría de Medio Ambiente y Recursos Naturales [Semarnat], 2015). El sector Uscusys incluye las emisiones y absorciones asociadas a los cambios de uso del suelo de tierras forestales, pastizales, tierras agrícolas, humedales, asentamientos humanos y otras tierras (IneccSemarnat, 2015) y, según la información obtenida en Inegei's previos (Inecc-Semarnat, 2012; INE-Semarnat, 2009; INE-Semarnat, 2006), sus emisiones estarían disminuyendo con el tiempo (en 2002, 89854 Gg; 14.0\% de las emisiones totales; INE-Semarnat, 2006). Estos datos, sin embargo, se deben tomar con cautela, ya que a lo largo de los años se han utilizado diferentes métodos de cálculo (Saynes, Etchevers, Paz y Alvarado, 2016).

Por otra parte, a escala estatal, la contribución relativa del cambio de uso del suelo y el sector forestal a las emisiones locales puede variar, destacándose los estados de Campeche, Chiapas y Tabasco, donde estos sectores llegaron a constituir 79\% (sector Uscusys en 2005; SemarnatCam, 2015), 59\% (sector Uscusys en 2005; Secretaría de Medio Ambiente, Vivienda e Historia Natural de Chiapas [Semavi], 2011) y $49.5 \%$ (Sectores Silvicultura y Uso de Suelo en periodo 2005-2007; Secretaría de Recursos
Naturales y Protección Ambiental del Estado de Tabasco [Sernapam], 2011) de las emisiones totales estatales, respectivamente.

La disminución de la deforestación y el aumento de los reservorios de carbono forestal han sido identificadas como medidas críticas y costo-efectivas para disminuir las emisiones globales y, a su vez, apoyar a los países en vías de desarrollo en la reducción de la pobreza y conservación de la biodiversidad y los servicios ecosistémicos (Eliasch, 2008). Según este informe, para reducir las emisiones del sector forestal a la mitad en 2030 se necesitan alrededor de USD $17 \times 10^{9}$ y USD $33 \times 10^{9}$ anuales, si se incluyen en esquemas de mercado, pero los beneficios netos podrían alcanzar los USD $3.7 \times 10^{12}$ en el largo plazo. El informe Stern (2007), a su vez, también calificó la reducción de la deforestación como un medio altamente rentable para reducir las emisiones de gases de efecto invernadero.

La respuesta política internacional al cambio climático comenzó con la adopción de la Convención Marco de las Naciones Unidas sobre el Cambio Climático [Cmnucc] en 1992. Esta convención establece un marco para la acción cuyo objetivo es la estabilización de la concentración de gases de efecto invernadero en la atmósfera, para evitar que interfiera peligrosamente con el sistema climático (Eguren, 2004). La Cmnucc entró en vigor el 21 de marzo de 1994 y actualmente está ratificada por 197 partes o países (Cmnucc, 2014a). De manera general, los dos grandes logros de la Cmnucc en la lucha contra el cambio climático han sido el alcance de dos acuerdos globales: el Protocolo de Kioto (adoptado en 1997) y el Acuerdo de París (adoptado en 2015).

De las negociaciones y acuerdos alcanzados en el seno de la Cmnucc han surgido mecanismos e iniciativas encaminadas a disminuir las emisiones asociadas al sector forestal, aunque el sector privado, organizaciones sin fines de lucro, gobiernos nacionales y subnacionales e iniciativas locales han aportado ideas y acciones a esta tarea global, las cuales han retroalimentado las discusiones y mecanismos impulsados en la Cmnucc.

Los distintos tipos de costos que enfrentan este tipo de proyectos e iniciativas en sus distintas fases (e.g., costos 
de implementación, transacción, oportunidad), así como la duración de los mismos (varias décadas), plantean retos para su financiamiento, el cual puede clasificarse, de manera general, en: a) financiamiento inicial y b) pagos basados en resultados. El primero suele cubrir distintas actividades relacionadas con la implementación del proyecto o iniciativa, así como algunos costos de transacción; en este caso, el financiamiento de fuentes privadas ha sido clave (Peters-Stanley, González y Yin, 2013). Los pagos basados en resultados se centran en la compra de los créditos de carbono generados por el proyecto, los cuales pueden, a su vez, cubrir algunos costos de transacción. La compra-venta de créditos de carbono se ha organizado, principalmente, en torno a mecanismos de mercado, aunque también se han desarrollado otros enfoques (World Bank, 2016; Cmnucc, 2017).

La comprensión del funcionamiento de los diferentes mecanismos de financiación que se han utilizado para mitigar las emisiones del sector forestal es fundamental para plantear esquemas efectivos capaces de abarcar grandes escalas y alcanzar el cumplimiento de objetivos nacionales de mitigación. Por ello, en este trabajo se presenta una síntesis de los mecanismos implementados a escala global y en distintos países y regiones del mundo, además de las experiencias desarrolladas en México. Con base en esta información se analizaron las oportunidades de incluir el sector forestal o potenciar su papel en los mecanismos financieros para la mitigación de emisiones que existen o están en desarrollo en el país.

\section{MATERIALES Y MÉTODOS}

La información sobre las distintas experiencias desarrolladas a escala internacional y nacional procede de una exhaustiva búsqueda bibliográfica de documentos de diversa índole: documentos oficiales de distintos gobiernos y organismos internacionales, reportes técnicos, páginas web de fondos climáticos, mecanismos de mercado y estándares de carbono, entre otros. En varias ocasiones se generaron cuadros resumen con datos de diferentes fuentes, para facilitar la visualización y comprensión de la información.
Los resultados del trabajo se estructuran en dos grandes secciones: las experiencias internacionales y las experiencias nacionales. Las experiencias internacionales se dividen, a su vez, en: a) los mecanismos de flexibilidad del Protocolo de Kioto; b) los nuevos mecanismos de mitigación surgidos a partir del Plan de Acción de Bali; y c) los mercados voluntarios de carbono. En la sección sobre las experiencias en México se presentan, en primer lugar, los distintos tipos de proyectos e iniciativas que se han desarrollado en el país, seguido de un apartado en el que se analizan las oportunidades de mitigación en el sector Uscusys de México. Por último, se incluye un apartado de conclusiones.

\section{Experiencias internacionales}

\section{El Protocolo de Kioto y sus mecanismos de flexibilidad} En la Conferencia de las Partes [COP] de la Cmnucc celebrada en Kioto (Japón) en diciembre de 1997 (COP 3), se adoptó el Protocolo de Kioto (PK) que entró en operación en 2005 y constituyó un primer esfuerzo para controlar los gases de efecto invernadero (GEI), ofreciendo un marco básico de acción para la lucha contra el cambio climático a escala internacional. El Protocolo contiene metas vinculantes de reducción y limitación de emisiones de gases de efecto invernadero para 37 países industrializados y la Comunidad Europea.

Conjuntamente, las emisiones de los seis gases incluidos bajo su control $\left(\mathrm{CO}_{2}, \mathrm{CH}_{4}, \mathrm{~N}_{2} \mathrm{O}, \mathrm{HFC}, \mathrm{PFC}, \mathrm{SF}_{6}\right)$ deberían reducirse en, al menos, 5\% hacia 2012 sobre la base de las emisiones que se registraban en 1990. Con excepción de los Estados Unidos, todas las Partes que son países altamente industrializados ratificaron el PK y, según la Cmnucc (2015a), en 2012, las emisiones de los países con reducciones de GEI obligatorias bajo el PK se habían reducido en $22.6 \%$ con respecto al año base, aunque este logro no es enteramente atribuido a la acción del PK. Según Puigdueta y Sanz (2017), la disminución de las emisiones de GEI podría haberse visto favorecida por otros hechos, como el colapso de la Unión Soviética y el consecuente impacto en su economía, la relocalización de 
industrias altamente contaminantes hacia países que no tenían obligaciones de reducción bajo el Protocolo o la crisis financiera mundial de 2008.

Tras la conclusión del primer periodo de compromiso del PK en 2012, se ratificó un segundo periodo en la COP 18 de Doha, en Qatar, con vigencia entre 2013 y 2020; sin embargo, el grupo de países firmantes fue menor. En este nuevo periodo del PK, los países firmantes se comprometieron a reducir el total de sus emisiones a un nivel inferior en no menos de $18 \%$ con respecto al de 1990 , en el periodo de compromiso comprendido entre los años 2013 y 2020. En este periodo se incluyó un nuevo gas de efecto invernadero: el Trifluoruro de nitrógeno $\left(\mathrm{NF}_{3}\right)$ (Cmnucc, 2012).

El PK incluye tres mecanismos de flexibilidad para facilitar a los países del Anexo I de la Convención Marco de Naciones sobre el Cambio Climático (países desarrollados y con economías en transición hacia economías de mercado) la consecución de sus objetivos de reducción y limitación de emisiones de GEI. Estos tres mecanismos son: el comercio de derechos de emisión, la aplicación conjunta y el mecanismo para un desarrollo limpio [MDL].

\section{El comercio de emisiones}

El PK permitió el comercio de los derechos de emisión (mercado de carbono) entre las Partes, bajo el supuesto de que toda operación de este tipo es suplementaria a las medidas nacionales que se adopten para cumplir los compromisos cuantitativos de limitación y reducción de emisiones. Ante este régimen, los países Partes del Anexo I, o aquellas personas jurídicas a las que éstos hayan autorizado, pueden intercambiar en el mercado los distintos tipos de unidades contables reconocidas por el Protocolo: 1) Unidades de Cantidad Atribuida inicialmente asignadas a cada Parte [UCAs]; 2) Unidades de Reducción de Emisiones de proyectos de aplicación conjunta [UREs]; 3) Reducciones Certificadas de Emisiones generadas por proyectos del Mecanismo de Desarrollo Limpio [RCEs, por sus siglas en inglés]; 4) Unidades de Absorción procedentes de actividades en sumideros [UDAs].

Este mecanismo ha operado generalmente bajo esquemas de cap and trade o tope y canje, en donde los gobier- nos imponen límites a las emisiones de los sectores incluidos en la regulación (Seeberg-Elverfeldt, 2010). Las empresas, industrias o afectados tienen que adquirir permisos de emisión que representan el derecho a emitir un volumen específico de GEI; de esta forma, las empresas que necesitan aumentar su volumen de emisiones deben comprar permisos a otros que requieren menos, creando un mercado regulado que permite disminuir las emisiones y que, además, incentiva las buenas prácticas para crear una economía baja en carbono.

En consecuencia, a escala global surgieron diferentes esquemas de comercio de emisiones, siendo el principal el mercado europeo.

\section{El mercado Europeo}

El Mercado Europeo de Derechos de Emisión [EU-ETS por sus siglas en inglés] surgió en 2005 y fue el primer mercado de carbono regional (actualmente participan 31 países) y es el principal mercado de carbono mundial; además, es el principal demandante de RCEs y el que determina, en gran medida, los precios de estos certificados (European Comission, 2017a). Actualmente, el mercado se encuentra en su tercera fase (periodo 2013-2020) y no admite créditos provenientes de proyectos forestales.

La propuesta planteada en el Marco Europeo para el Clima y la Energía en el periodo 2021-2030, sin embargo, considera el sector Uscusys como uno de sus pilares, bajo la denominada "no debits rule", la cual consiste en que cada estado miembro debe asegurarse de que las emisiones provenientes de este sector sean enteramente compensadas por las remociones asociadas a actividades adicionales emprendidas en el sector (European Comission, 2017b), aunque se planea admitir cierta flexibilidad.

\section{Los proyectos forestales en los esquemas de comercio de emisiones}

Los principales mercados regulados que han admitido o admiten créditos de proyectos forestales han sido los desarrollados en Nueva Gales del Sur en Australia (New South Wales Greenhouse Gas Abatment Scheme o NSW GGAS), Australia, Nueva Zelanda (Tabla 1). 
Desde 2001, Australia ha probado diferentes esquemas de mercado de emisiones a escala estatal y también nacional (Díaz, Hamilton y Johson, 2011). El NSW GGAS fue el primer esquema regulado de comercio de emisiones a escala mundial, operó a escala estatal y estuvo activo entre 2003 y 2012, cuando en Australia entró en vigor su Mecanismo de Fijación de Precio del Carbono (Carbon Pricing Mechanism, por sus siglas en inglés). Este mecanismo cubría aproximadamente $60 \%$ de sus emisiones (sector eléctrico) y buscaba llegar a un esquema de cap and trade por fases, comenzando con un precio fijo del carbono durante los tres primeros años (Center for Climate and Energy Solutions, 2011). Como parte de esta política se implementó un programa nacional de compensaciones llamado Carbon Farming Initiative (CFI), que permitía a los granjeros y administradores de la tierra ganar créditos de carbono a través del almacenamiento o reducción de emisiones de GEI. Esta iniciativa incluía proyectos relacionados con quemas controladas en sabanas y actividades de reforestación. Los créditos generados podían ser vendidos a personas o negocios que quisieran compensar sus emisiones (Australian Government, 2017a).

Posteriormente, en 2014, Australia cambió su política de reducción de emisiones y creó su Fondo de Reducción de Emisiones (Emission Reduction Fund), con el objetivo de ayudar al país a alcanzar sus metas de reduc- ción de emisiones en 2020. El gobierno le asignó un presupuesto inicial de USD $1.94 \times 10^{9} \mathrm{y}$ su financiamiento debe ser considerado en los futuros presupuestos (Australian Government, 2017b). Este fondo admite proyectos del sector agrícola (manejo de ganado, reducción de emisiones de metano, uso eficiente de fertilizantes y captura de carbono en suelos de pastizal, entre otros) y manejo de la vegetación (deforestación evitada, plantación de árboles, quemas controladas en sabanas, etc.). A través de este

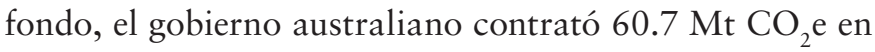
créditos de carbono provenientes de proyectos relacionados con el uso del suelo, por un valor total de USD $588 \times$ $10^{6}$ (Goldstein y Ruef, 2016).

Nueva Zelanda cuenta con un Esquema de Comercio de Emisiones (NZ ETS) desde 2008 en el que el sector forestal fue el único regulado hasta el 2010, cuando los sectores industria, transporte y energía fueron también incluidos (Díaz et al., 2011).

La política climática de Nueva Zelanda admite dos esquemas de participación para los bosques establecidos después de 1989, los cuales pueden emitir créditos en el marco del NW ETS o pueden participar en la Permanent Forest Sink Initiative (PFSI), comprometiéndose a mantener la cobertura forestal en el terreno durante 99 años (Díaz et al., 2011). Los créditos emitidos a través de la PFSI han alcanzado precios hasta $25 \%$ más elevados fuera

Tabla 1. Mercados regulados en los que han participado proyectos forestales y relacionados con el uso de la tierra.

\begin{tabular}{|c|c|c|c|c|c|c|}
\hline Mercado & Tiempo & $\begin{array}{l}\text { Vol. histórico } \\
\left(\mathrm{MtCO}_{2} e\right)\end{array}$ & $\begin{array}{l}\text { Valor histórico } \\
\left(U S D \times 10^{6}\right)\end{array}$ & $\begin{array}{l}\text { Precio promedio/intervalo } \\
\text { de precios }\left(\mathrm{USD} / \mathrm{CO}_{2} e\right)\end{array}$ & $\begin{array}{l}\text { Tipos de } \\
\text { proyectos }\end{array}$ & $\begin{array}{c}\text { Créditos internacio- } \\
\text { nales aceptados }\end{array}$ \\
\hline NSW & 2003-2012 & 6.3 & S.D. & $0.15-15$ & $A / R^{*}$ & No \\
\hline CFI-Australia & $2012-2014$ & 8.4 & 141 & 17.3 & $\begin{array}{c}\text { Quema sabanas, } \\
\text { A/R }\end{array}$ & No \\
\hline ERF-Australia & $2014-2015$ & 60.7 & 588 & 9.7 & $\begin{array}{l}\text { Agrícolas y } \\
\text { Forestales }\end{array}$ & No \\
\hline$N Z$ & 2008-2015 & 2.3 & 21.1 & 13.9-3.1 & $\begin{array}{c}\text { Reforestación/ } \\
\text { deforestación } \\
\text { evitada }\end{array}$ & $\begin{array}{l}\text { Si, hasta } 2015 \\
\text { (MDL) }\end{array}$ \\
\hline
\end{tabular}

Fuente: Elaboración propia, con datos de Goldstein y Neyland (2015), Goldstein y Ruef (2016) e IPART (2012). *A/R: Forestación/reforestación (por sus siglas en inglés). 
del mercado nacional, ya que se considera que sus cobeneficios son mayores (Nabuurs et al., 2015).

Inicialmente el esquema admitía créditos de compensaciones internacionales; sin embargo, la caída de los precios de los créditos a consecuencia de la aceptación ilimitada de créditos de compensación de proyectos MDL ubicados en otros países (precio promedio en 2010: NZD 20/t $\mathrm{CO}_{2}$ e; precio promedio en 2013: NZD 1.50/t $\mathrm{CO}_{2} \mathrm{e}$; Hamrick, 2015) llevó al gobierno, en 2015, a no permitir la utilización de unidades internacionales en el esquema (Goldstein y Ruef, 2016).

En 2016, el país concluyó una revisión del sistema con el fin de identificar las adaptaciones necesarias para que sea un mecanismo eficiente para alcanzar sus compromisos en 2030 en el marco del Acuerdo de París. El resultado de la revisión propone algunas reformas internas y resalta el interés por ligar el mercado nacional con otros mercados, aunque se reconoce que bajo el Acuerdo de París será necesario llegar a acuerdos bilaterales y multilaterales con otros países para lograr el acceso a unidades de reducción de emisiones internacionales de alta calidad (New Zealand Ministry for the Environment, 2017).

\section{La Aplicación Conjunta}

Este mecanismo permite que un país del Anexo I invierta en proyectos de reducción de emisiones o captura de carbono desarrollados en otro país del Anexo I, de tal forma que el país inversor adquiere unidades de reducción de emisiones que puede utilizar para el logro de sus metas asociadas al PK (Cmnucc, 2014b). Al mismo tiempo, la Parte donde se lleva a cabo el proyecto se beneficia de la inversión extranjera y la transferencia de tecnología.

\section{El Mecanismo para un Desarrollo Limpio}

El MDL fue el mayor y más activo programa de compensaciones de emisiones de cumplimiento a escala mundial, fuente de créditos de compensación para los países firmantes del PK y compradores del mercado europeo (Cmnucc, 2014c). Este programa es, de hecho, el único de los mecanismos de flexibilidad en el que pueden participar los países en desarrollo o No Anexo I (Seeberg-Elverfeldt,
2010). Adicionalmente, el MDL busca que el país receptor de la inversión consiga un desarrollo sostenible a través de la transferencia de tecnologías limpias y, a su vez, contribuya a alcanzar el objetivo último de la Cmnucc.

Globalmente, donde se han desarrollado más proyectos del MDL ha sido la región Asia-Pacífico que alberga $82 \%$ del total, seguida de América Latina con 13\%. Dentro de América Latina, Brasil ha hospedado mayor número de proyectos (35\%), seguido de México (18\%) (UNEP DTU Partnership, 2017a).

Los únicos proyectos relacionados con el sector forestal contemplados en el mecanismo son los proyectos de forestación/reforestación (A/R, por sus siglas en inglés), que constituyen únicamente $0.8 \%$ del total (UNEP DTU Partnership, 2017b), frente a los proyectos del sector de las energías renovables que abarca $71 \%$ de los proyectos MDL. Las razones del escaso número de proyectos $A / R$ implementados se asocian con diversas causas, entre las que destacan la complejidad de la regulación para su implementación, el mayor riesgo financiero asociado a este tipo de proyectos (permanencia, precisión del monitoreo), la tardanza en generar créditos de carbono, su exclusión del mercado europeo y la estructura temporal de los créditos (Hamilton, Sjardin, Peters-Stanley y Marcello, 2010), con certificados de reducción de emisiones temporales o tRCEs (que se expiden por cinco años) y certificados de reducción de emisiones de largo plazo o lRCEs (se expiden por 20 años con oportunidad de renovarse por otros 20 años más o de 30 años sin oportunidad de renovarse).

Los precios de los RCEs se mantuvieron por encima de los USD 10/t $\mathrm{CO}_{2}$ e hasta 2009, pero en 2012 su precio promedio se situó en USD 3.2/t $\mathrm{CO}_{2}$ e (Peters-Stanley et al., 2013) y en 2016, en USD 1.6/t $\mathrm{CO}_{2}$ e (Hamrick y Gallant, 2017a). Por su parte, los RCEs de proyectos A/R alcanzaron su mejor precio en 2007 con USD 5.7/t $\mathrm{CO}_{2} \mathrm{e}$ (Hamilton et al., 2010) pero en 2012 su precio había bajado a USD 1.1/t $\mathrm{CO}_{2}$ e (Goldstein y González, 2014). En conjunto, los proyectos MDL han expedido más de 1800 millones de RCEs (Cmnucc, 2016a), correspondiendo más de 11.3 millones a proyectos A/R. Sin 
embargo, tras la finalización del primer periodo de compromiso del PK, la demanda de RCEs disminuyó considerablemente debido, entre otras cosas, al menor número de partes que participan en el segundo periodo de compromiso, las metas de emisión que los países se han comprometido a alcanzar, las restricciones que algunos compradores aplican con respecto al tipo y el origen de los RCEs y la prolongada recesión económica que afectó a varios países desarrollados (Cmnucc, 2013).

Como consecuencia, muchos proyectos han dejado de expedir RCEs; de hecho, se calcula que aproximadamente $42 \%$ de los proyectos que expidieron RCEs hasta el final del primer periodo del PK no lo han hecho en el segundo periodo (Cmnucc, 2016a). Además, existe una incertidumbre considerable acerca de si el MDL se utilizará -y de qué manera- después del 2020. Aun así, el MDL ha demostrado su potencial como instrumento para movilizar fondos destinados a la acción climática y el desarrollo sostenible (Cmnucc, 2016a).

\section{Los nuevos mecanismos de mitigación: del Plan} de Acción de Bali al Acuerdo de París

En un proceso paralelo al PK, a partir de la COP 13 celebrada en Bali en 2007, la Cmnucc estableció un plan de acción para facilitar la transición a una segunda fase de la Convención tras el 2012. El Plan de Acción de Bali (decisión 1/CP.13), sentó las bases para la cooperación a largo plazo entre las partes y marcó la trayectoria de un nuevo proceso de negociación diseñado para hacer frente al cambio climático, en el que el papel de los países en vías de desarrollo cobra mayor relevancia.

En este contexto surgieron nuevos mecanismos de mitigación acordes con los nuevos retos identificados y la necesidad de actuar a escalas mayores a la de proyecto, con el fin de asegurar la permanencia de las acciones emprendidas y evitar las fugas. Entre estos, destaca el mecanismo de Reducción de Emisiones por Deforestación y Degradación forestal más la conservación, el manejo forestal sostenible y el incremento de los almacenes forestales (REDD+) que se centra en reducir las emisiones del sector forestal en países en vías de desarrollo conside- rando como escala de actuación un país o una jurisdicción al completo. Las Acciones de Mitigación Nacionalmente Apropiadas (NAMAs, por sus siglas en inglés) se enfocan en acciones sectoriales que pueden incluir al sector Afous. Adicionalmente, las estrategias de desarrollo bajo en emisiones (LEDS, por sus siglas en inglés) son visualizadas como el marco amplio en el que se pueden insertar acciones más concretas como las NAMAs o políticas o enfoques relacionados con REDD+.

La culminación del proceso iniciado en Bali llegó con el Acuerdo de París en 2015 (COP 21), que logró establecer un nuevo compromiso global por el clima, cuyos rasgos más distintivos son: a) establece compromisos globales y no sólo compromisos para los países del Anexo I; b) es flexible para cada una de las Partes, ya que cada país propondrá sus Contribuciones Determinadas a Nivel Nacional (NDCs, por sus siglas en inglés); c) amplía sus acciones a mitigación y adaptación, reforzando las acciones contra el cambio climático en el contexto del desarrollo sostenible y los esfuerzos por erradicar la pobreza (Artículo 2.1.); d) alienta el desarrollo de capacidades y la transferencia tecnológica, para mejorar la resiliencia al cambio climático y reducir las emisiones de gases de efecto invernadero; e) establece que los países desarrollados deberán proporcionar recursos financieros a las Partes que son países en desarrollo para prestarles asistencia tanto en la mitigación como en la adaptación (Artículo 9.1.) (Cmnucc, 2015b).

El papel de los bosques como sumideros y reservorios de carbono es reconocido en el Artículo 5 del Acuerdo, donde se alienta a las Partes a adoptar enfoques de política e incentivos positivos para REDD+, mediante pagos basados en resultados (Cmnucc, 2015b). Adicionalmente, en su Artículo 4, el Acuerdo insta a las partes a formular sus LEDs a largo plazo, tomando en cuenta las responsabilidades comunes pero diferenciadas. Las NAMAs, por su parte, no aparecen nombradas en el Acuerdo aunque sí se hace referencia a la necesidad de implementar acciones de mitigación.

El desarrollo de estos nuevos mecanismos de mitigación de emisiones ha supuesto la necesidad de ampliar los enfoques para su financiamiento, más allá de los mecanis- 
mos del PK. De hecho, siguiendo el mandato de la COP de Bali en 2007, las Partes propusieron establecer un Nuevo Mecanismo de Mercado (NMM) durante la COP 17 en Durban (2011), confiando esta labor al SBSTA (Subsidiary Body For Scientific and Technological Advice) de la Cmnucc, el cual también está desarrollando otros tipos de instrumentos económicos (Tabla 2) y es el encargado de desarrollar y elaborar las recomendaciones para asegurar una contabilidad robusta en el marco de los enfoques cooperativos.

El Acuerdo de París busca acelerar y ampliar la disponibilidad de recursos financieros para la acción climática. Institucionalmente, el Fondo Verde para el Clima (GCF, por sus siglas en inglés) y el Fondo para el Medio Ambiente Mundial (GEF, por sus siglas en inglés) serán las entidades encargadas del mecanismo financiero de la Convención y servirán también como mecanismo financiero del Acuerdo. Otro aspecto importante es que el Acuerdo mantiene y establece la continuidad a futuro de los objetivos colecti-

TABLA 2. Instrumentos económicos en desarrollo de la Cmnucc.

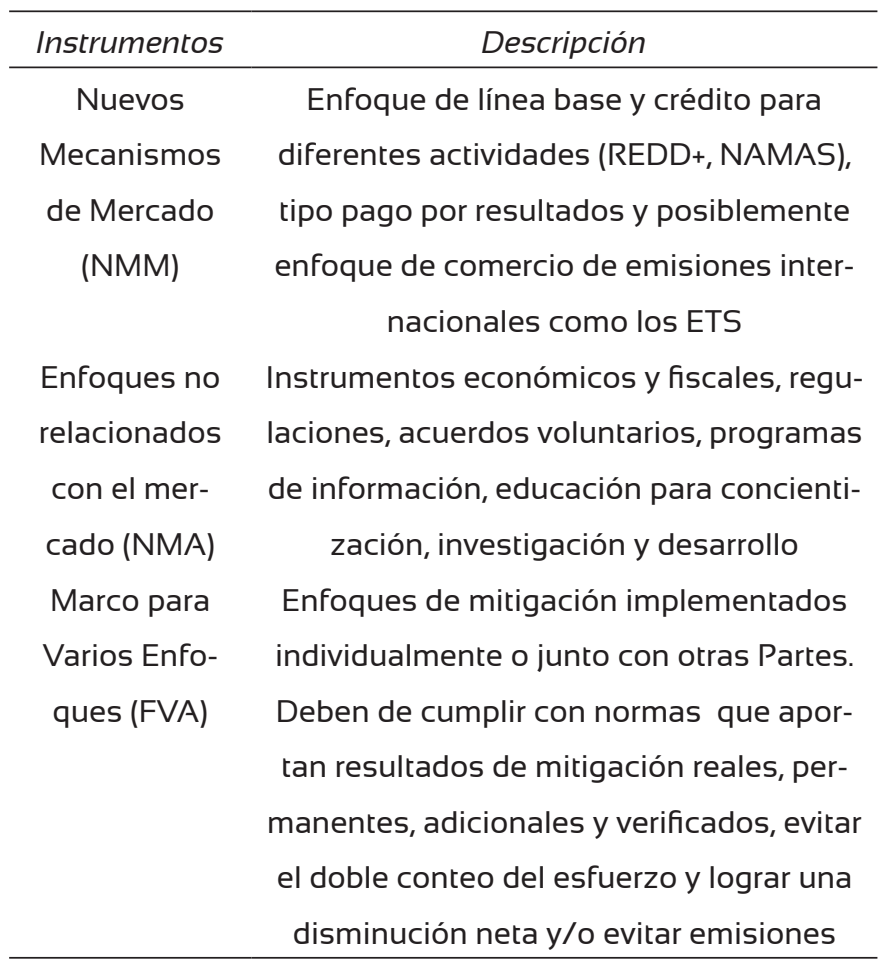

Fuente: Cmnucc (2017) vos cuantificados de financiamiento (mínimo 100000 millones de dólares anuales, teniendo en cuenta las necesidades y prioridades de los países en desarrollo). Para la consecución de los compromisos del Acuerdo, el financiamiento público tiene un rol crítico, en especial en las acciones destinadas a la mejora de la resiliencia y adaptación, y también en cuanto a que permite apalancar y movilizar recursos de otras fuentes; en particular, fondos del sector privado (Carlino, Netto, Cabrera y Serra 2017).

\section{Instrumentos de fijación del precio del carbono}

En los últimos años se ha notado un auge en el desarrollo e implementación de instrumentos de fijación del precio del carbono en diversas regiones, como consecuencia de la falta de acuerdo post Kioto en 2012 y la baja demanda de RCEs en el mercado. Los principales instrumentos utilizados han sido los sistemas de comercio de emisiones (ETS) y los impuestos al carbono, cuyo principal propósito ha sido dar a las empresas y consumidores un incentivo para usar fuentes alternativas de energía o usar menos energía. Estos instrumentos operan a escala de grupos de países, a escala nacional y a escala subnacional (abarcando una o varias regiones o estados), existiendo actualmente 39 iniciativas nacionales y 23 a escala subnacional (World Bank, 2016).

\section{Mercado de California}

Para México, tiene especial interés el esquema desarrollado en California que en 2006 aprobó la Global Warming Solutions Act (AB 32). En él se establece el diseño e implementación de un programa de cap and trade que abarca $85 \%$ de las emisiones del estado, y cuyo esquema incluyó la posibilidad de compensar hasta $8 \%$ de las emisiones a través de créditos de compensación de emisiones (Díaz et al., 2011). El programa está activo desde 2013 y la AB32 estableció su duración hasta el 2020. Sin embargo, en julio de 2017 fue aprobada la AB398 que extiende el esquema hasta el año 2030. La información relativa al volumen, valor de mercado y precios de los créditos de carbono se presenta en la tabla 3.

La Air Resources Board (ARB), entidad encargada de gestionar el esquema, ha aprobado seis protocolos de 
compensación de emisiones (CAR, 2017) para proyectos ubicados en Estados Unidos -uno de ellos sobre manejo forestal- y, hasta el momento, más de $70 \%$ de los créditos de compensación emitidos por la ARB provienen de proyectos forestales (ARB, 2017). Fue creado también un Protocolo Forestal para México (primera versión en 2013), que se enfoca en actividades que incrementan los acervos de carbono, aunque no puede ser utilizado para compensar emisiones en el mercado de California.

Hasta ahora no se han admitido créditos de compensación internacionales, aunque la $\mathrm{ARB}$ ha considerado la inclusión de créditos REDD+ en el esquema. En este sentido, destaca el memorándum de entendimiento firmado por California con los estados de Chiapas (México) y Acre (Brasil) a finales de 2010, para iniciar un proceso a fin de que puedan generar créditos REDD+ para el mercado de California, bajo esquemas jurisdiccionales y la creación del Grupo de trabajo de Compensaciones REDD+, que emitió sus recomendaciones en 2015.

Esta situación podría cambiar en los próximos años ya que el nuevo proyecto de ley abre la puerta a la utilización de créditos de compensación de emisiones internacionales tras el 2020, aunque el porcentaje de créditos de compensación permitidos disminuye $(4 \%$ de las emisiones pueden ser indemnizadas con créditos de compensación en el periodo 2020-2025 y 6\% en el periodo 2026-2030) y con la restricción de que al menos la mitad de estos créditos deben provenir de proyectos ubicados en California (ARB, 2017).

California forma parte de la Western Climate Initiative (WCI), una organización sin fines de lucro que promueve la creación de un esquema de mercado para reducir los GEI en América del Norte (Hamrick y Gallant, 2017b). Actualmente la WCI está formada por el estado de California y por tres provincias canadienses (Quebec, Ontario y British Columbia). En 2014, California y Quebec asociaron sus programas de cap and trade. Ampliando esta asociación, en septiembre de 2015, Ontario firmó un memorándum de entendimiento con Quebec para armonizar información y metodologías de inventario con Quebec y California, para instalar un sistema de créditos de compensación. A principios de 2017 Ontario lanzó su programa de cap and trade, el cual, desde septiembre de 2017, quedó oficialmente ligado al programa de CaliforniaQuebec (Hamrick y Gallant, 2017b).

\section{Mecanismo de reducción por deforestación y degradación forestal evitada}

Los conceptos asociados con el mecanismo REDD+ comenzaron a discutirse en el marco de la Cmnucc a partir de 2005, en la COP 11 de Montreal, cuando Costa Rica y Papua Nueva Guinea sometieron a las Partes una propuesta donde se expresaba la creciente concientización sobre la contribución de la deforestación a las emisiones de GEI; de esta forma, surge el término de deforestación evitada en el marco de la negociación.

El mecanismo REDD+ ha evolucionado en el transcurso de los años. En total, entre 2007 y 2015 se han tomado 16 decisiones claves que han estructurado el mecanismo REDD+ como una estrategia para desacelerar, detener y revertir la pérdida de carbono y de la cubierta forestal (Cmnucc, 2016b). El mecanismo se basa en el pago a países por su desempeño en la reducción de sus emisiones por deforestación y degradación forestal, la cual ha de ser

Tabla 3. Mercado de California.

\begin{tabular}{|c|c|c|c|c|c|c|}
\hline Mercado & Tiempo & $\begin{array}{l}\text { Vol. histórico } \\
(\mathrm{MrCO} e)\end{array}$ & $\begin{array}{l}\text { Valor histórico } \\
\left(U S D \times 10^{6}\right)\end{array}$ & $\begin{array}{l}\text { Precio promedio/intervalo } \\
\text { de precios (USD/t } \mathrm{CO}_{2} \text { e) }\end{array}$ & Tipos de proyectos & $\begin{array}{c}\text { Créditos internacio- } \\
\text { nales aceptados }\end{array}$ \\
\hline $\begin{array}{l}\text { California- } \\
\text { Quebec }\end{array}$ & 2013-2015 & 14.3 & 134 & 9.3 & $\begin{array}{c}\text { Manejo forestal } \\
\text { mejorado }\end{array}$ & No \\
\hline
\end{tabular}

Fuente: Elaboración propia, con datos de Goldstein y Neyland (2015), Goldstein y Ruef (2O16) y ARB (2017). 
cuantificada a escala nacional a través de un sistema robusto y transparente de monitoreo de los bosques.

Los Acuerdos de Cancún (Decisión 1/CP.16) fueron un hito importante para el mecanismo REDD+ ya que establecieron su ámbito de actuación y los principios y las salvaguardas a tener en cuenta en las estrategias y actividades REDD+. En esta decisión se plantea que los países que deseen implementar mecanismos REDD+ deben desarrollar cuatro elementos: 1) una estrategia nacional, 2) un nivel de referencia de las emisiones forestales, 3) un sistema de monitoreo, reporte y verificación y 4) un sistema de información de salvaguardas. Además, plantea que las actividades relacionadas con el mecanismo REDD+ se implementen por fases: 1) preparación (readiness) en la que se desarrollan estrategias nacionales y se realizan esfuerzos para la creación de capacidades; 2) implementación de las estrategias y medidas nacionales y 3) actividades basadas en pagos por resultados que deberían ser objeto de monitoreo, reporte y verificación.

La implementación de REDD+, supuso, por tanto, un gran reto para los países interesados en este mecanismo, ya que tuvieron que hacer ajustes en sus políticas nacionales, leyes, instituciones y crear capacidades relacionadas con temas clave, como las salvaguardas y el monitoreo de los bosques. El financiamiento requerido para implementar este tipo de mecanismos ha requerido, por tanto, la movilización de importantes recursos a escala nacional e internacional.
Hasta la fecha, la fase de preparación del mecanismo REDD+ se ha financiado a través de acuerdos bilaterales y agencias multilaterales. Según Goldstein y Ruef (2016), los únicos pagos basados en resultados para reducir emisiones relacionadas con los bosques las han realizado, el programa alemán REDD+ Early Movers (con aportaciones de Alemania, Noruega y Reino Unido) a Colombia y el estado brasileño de Acre y del gobierno de Noruega a Guyana (Tabla 4). Adicionalmente, el Fondo Amazonía (Brasil), creado con donaciones del gobierno de Noruega, Alemania y la compañía brasileña Petrobas, ha recibido pagos por la reducción anual de la deforestación en el país. El Fondo Amazonía y el programa REDD+ Early Movers han firmado acuerdos con un precio de USD 5/t $\mathrm{CO}_{2} \mathrm{e}$ (Goldstein y Ruef, 2016), aunque este valor no debe considerarse un valor de mercado.

En los próximos años se espera que el financiamiento basado en pagos por resultados cobre mayor relevancia, dado que el Programa REDD+ Early Movers sigue activo en Acre y en Colombia y, adicionalmente, tiene firmado un memorándum de entendimiento con Ecuador. El gobierno de Noruega, por su parte, tiene acuerdos bilaterales con la República Democrática del Congo, Indonesia, Liberia y Perú, que todavía no se han traducido en pagos, además de los procesos activos con Colombia y Guyana. Existen, además, otras iniciativas como el BioCarbon Fund Initiative for Sustainable Forest Landscapes (ISFL) que ha firmado una carta de intención con una región de

TABla 4. Pagos basados en resultados para REDD+.

\begin{tabular}{|c|c|c|c|c|}
\hline Donante & $\begin{array}{c}\text { País/estado } \\
\text { receptor }\end{array}$ & $\begin{array}{c}\text { Instrumento financiero/ } \\
\text { Programa }\end{array}$ & $\begin{array}{c}\text { Monto recibido hasta } \\
2017\left(\mathrm{USD} \times 10_{6}\right)\end{array}$ & $\begin{array}{l}\text { Emisiones reducidas } \\
\qquad\left(\mathrm{MrCO} \mathrm{CO}_{2} e\right)\end{array}$ \\
\hline Agencia Alemana de & Acre (Brasil) & REDD early movers & 36 & 8 \\
\hline Cooperación & Colombia & REDD early movers & 27 & 20 \\
\hline Noruega & Guyana & Guyana REDD+ investment fund & 70 & \\
\hline
\end{tabular}

Fuente: Elaboración propia con datos del Amazon Fund (2017), GRIF (2015), Goldstein y Ruef (2016) y Hamrick y Gallant (2Ol7b)

*Monto hasta 2016. 
Etiopía y está prevista la firma de dos cartas de intención más con regiones de Zambia y Colombia (Hamrick y Gallant, 2017b).

El Forest Carbon Partnership Facility (FCPF) es una alianza global que apoya la preparación e implementación de mecanismos REDD+ en países con bosques tropicales y subtropicales. El FCPF entró en operación en 2008 y cuenta con dos mecanismos de financiamiento complementarios: 1) el Fondo de Preparación y 2) el Fondo de Carbono. Los donantes de ambos fondos son entidades gubernamentales, no gubernamentales y compañías privadas (FCPF, 2017). Una vez que los países incluidos en el programa han avanzado suficientemente en su fase de preparación pueden ser seleccionados para participar en el Fondo de Carbono y recibir pagos por resultados de actividades piloto. El FCPF ha apoyado a 47 países en su proceso de preparación y se espera que algunos de ellos firmen pronto Acuerdos de Pago por Reducción de Emisiones (Erpas, por sus siglas en inglés) ya que Chile, la República Democrática del Congo y Ghana han sido oficialmente incluidos en el portafolio del Fondo de Carbono y, probablemente serán los primeros en firmar Erpas. Adicionalmente, Costa Rica, México y la República del Congo han sido provisionalmente aceptados, aunque tienen pendiente algún requerimiento (Hamrick y Gallant, 2017b).

En 2017, el Fondo Verde para el Clima anunció un programa piloto para el pago por resultados asociados a REDD+ con un presupuesto de USD $300 \times 10^{6}$ a USD 500 $\times 10^{6}$, con un precio de USD 5/t $\mathrm{tC}_{2} \mathrm{e}$ (que podría incrementarse en caso de acreditarse beneficios adicionales al carbono) y para reducciones que se acrediten ente 2014 y 2018 (GCF, 2017). Además, los fondos prometidos por los países tras el Acuerdo de Paris, según Goldstein y Ruef (2016), podrían incrementar notablemente el financiamiento disponible para pagos por resultados asociados a REDD+.

Por otra parte, hasta el momento, los créditos REDD+ sólo son admitidos en el sistema colombiano, el cual se basa en un impuesto a las emisiones de GEI por el uso de combustibles fósiles, que permite utilizar créditos de compensación de diferentes tipos de proyectos, entre ellos pro- yectos REDD+, todos ellos basados en Colombia (Hamrick y Gallant, 2017b). Otros mercados regulados, como el de California, Corea del Sur y el sector de la aviación han expresado su interés en considerar créditos de compensación REDD+ procedentes de programas nacionales y jurisdiccionales (Hamrick y Gallant, 2017a).

\section{Acciones de mitigación nacionalmente apropiadas}

En el Plan de Acción de Bali (Cmnucc, 2007) en el Párrafo 1 (b) (ii) se hizo un llamado para llevar a cabo "acciones nacionales apropiadas de mitigación por los países en desarrollo en el contexto del desarrollo sostenible, soportadas y activadas por tecnología, financiamiento y construcción de capacidades, en una forma medible, reportable y verificable". Según su fuente de financiamiento, las NAMAs se clasifican en: a) unilaterales (sin el apoyo de terceros); b) apoyadas (con apoyo financiero, transferencia tecnológica y/o desarrollo de capacidades) y c) acreditadas (las reducciones son vendidas en el mercado de carbono).

En la COP 18 de Doha (2012) se lanzaron dos iniciativas con el fin de avanzar hacia las buenas prácticas para la financiación de las NAMAs: la NAMA facility y la NAMA partnership.

La NAMA facility es un fondo lanzado por el Ministerio de Medio Ambiente de Alemania y el Departamento de Negocios, Energía y Estrategia Industrial del Reino Unido, con el fin de apoyar a gobiernos de países en desarrollo que muestren su liderazgo en el tema de cambio climático para implementar NAMAs a través de apoyo financiero y técnico en diferentes sectores. El fondo busca que se produzcan transformaciones capaces de cambiar tecnologías o sectores completos hacia un desarrollo bajo en carbono (Agarwal et al., 2013). Este fondo abrió su primera convocatoria en 2013 con 69 millones de euros, posteriormente se sumaron agencias del Gobierno de Dinamarca y la Comisión Europea como donantes, lo que ha permitido lanzar cinco convocatorias y apoyar a 22 NAMAs en todo el mundo (NAMA facility, 2017).

Los proyectos apoyados por la NAMA facility abarcan, de manera general, los sectores de Eficiencia Energética, Transporte, Energía Renovable, Agricultura, 
Silvicultura y Residuos. En su portafolio se encuentran dos proyectos forestales en fase de implementación (Tabla $5)$.

Por otra parte, la NAMA partnership tiene el propósito de incentivar la colaboración y complementariedad de actividades de organismos multilaterales, bilaterales y otras organizaciones para compartir información y crear conocimiento técnico para acelerar el apoyo a los países en desarrollo en la preparación e implementación de NAMAs (Agarwal et al., 2013).

\section{Estrategias de desarrollo bajo en emisiones}

El término surgió por primera vez en 2008 en el marco de las negociaciones de la Cmnucc y, desde los Acuerdos de Cancún (2010), se animaba a las partes a la elaboración de LEDs en un contexto de desarrollo sostenible. Las LEDs tienen como objetivo ayudar a los países en la planificación de su ruta hacia un desarrollo bajo en emisiones, constituyéndose como un marco amplio, que abarca los sectores económicos más relevantes de un país y en el que puede insertarse el desarrollo de NAMAs.

Actualmente no existe una fuente de financiamiento específica para que los países desarrollen sus LEDs y se considera que éste debe proceder de diversas fuentes, incluyendo los recursos nacionales, para conseguir una mezcla efectiva de instrumentos políticos y económicos. En relación al fortalecimiento de capacidades, la Unión Europea y el PNUD (Programa de Desarrollo de las Naciones Unidas) crearon el Programa de Fortalecimiento de Capacidades en Bajas Emisiones (LECBP, por sus siglas en inglés) en 2011. Este programa busca fortalecer las capacidades a escala de país, al mismo tiempo que facili- tar la inclusión y coordinación de los sectores público y privado en iniciativas nacionales que buscan combatir el cambio climático (LECBP, 2017).

\section{Mercados voluntarios de carbono}

Los mercados voluntarios surgen a partir de la demanda voluntaria de créditos de carbono por parte de compañías e individuos que buscan compensar sus propias emisiones $\mathrm{y}$, también, de entidades que buscan comprar créditos de "precumplimiento", antes de que surjan o entren en vigor normas que regulen la necesidad de reducción de emisiones por parte de las empresas (Peters-Stanley y Yin, 2013). Según estos mismos autores, entre las motivaciones de los compradores voluntarios se encuentran la responsabilidad social corporativa, razones éticas, de reputación o temas como el riesgo en la cadena de suministro de las entidades. Por su parte, los compradores de créditos de "pre-cumplimiento" buscan conseguir créditos antes de la fecha de inicio de mercados regulados, con el fin de obtener un menor precio.

Las primeras transacciones que se hicieron en los mercados voluntarios datan de inicios de los 90 y los primeros proyectos de carbono que se implementaron fueron proyectos forestales (Hamilton et al., 2010). A pesar de ello, este tipo de proyectos jugó un papel marginal en los mercados regulados que surgieron a partir del PK, de hecho, según Goldstein y Ruef (2016), los proyectos de carbono forestal se han financiado históricamente a través de los mercados voluntarios, hasta el año 2015, en el que el Fondo Australiano de Reducción de Emisiones acaparó la mayor cuota de mercado.

La mayoría de las transacciones de créditos de carbono en el mercado voluntario se realizan a través de un

Tabla 5. Acciones de Mitigación Nacionalmente Apropiadas (NAMAs) en implementación en el sector forestal.

\begin{tabular}{cccccc}
\hline Sector & País & Proyecto & Financiador & Financiación & Mitigación \\
\hline Forestal & Tajikistán & NAMA forestal de Tajikistán & NAMA facility & EUR 13 $\times 10^{6}$ & $2.01 \mathrm{Mt} \mathrm{CO}_{2}$ e en 2O30 \\
Forestal & Georgia & $\begin{array}{c}\text { Manejo forestal sostenible adaptativo } \\
\text { en el Distrito de Borjomi-Bakuriani }\end{array}$ & $\begin{array}{c}\text { Ministerio austriaco } \\
\text { de Medioambiente }\end{array}$ & EUR $1.5 \times 10^{6}$ & Sin datos \\
\hline
\end{tabular}

Fuente: NAMA facility (2017) y Ecofys (2017) 
sistema descentralizado denominado over the counter (OTC) que consiste en realizar contratos bilaterales entre compradores y vendedores que definen los términos de pago y entregas (Peters-Stanley y Yin, 2013). Adicionalmente, se han desarrollado sistemas privados de intercambio, como fue el Chicago Climate Exchange que funcionó entre 2003 y 2010 como un sistema de cap and trade voluntario, con el fin de preparar a las empresas para futuros esquemas regulados a escala internacional, nacional y regional (Hamilton, Sjardin, Marcello y Gordon, 2008).

Los tipos de proyectos forestales que han participado en los mercados voluntarios de carbono son: proyectos $\mathrm{REDD}+$, proyectos $\mathrm{A} / \mathrm{R}$ y proyectos de manejo forestal mejorado (IFM, por sus siglas en inglés). Además, se han desarrollado proyectos agroforestales y de agricultura sostenible (suelos agrícolas y manejo de pastizales).

El volumen abarcado por los proyectos forestales ha variado entre $9 \%$ y $35 \%$ del volumen total de los mercados voluntarios de carbono, el cual ha oscilado entre 63 y

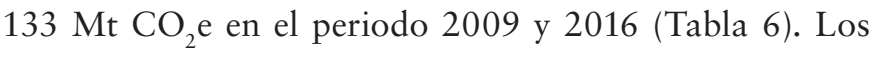
volúmenes del mercado muestran una tendencia a la baja, habiéndose situado en $63 \mathrm{Mt} \mathrm{CO}_{2}$ e en 2016 con un valor de mercado USD $74.2 \times 10^{6}$ (Hamrick y Gallant, 2017b).

TABla 6. Volumen total de los mercados voluntarios de carbono $\left(\mathrm{Mt} \mathrm{CO}_{2} \mathrm{e}\right)$ y de los proyectos forestales.

\begin{tabular}{ccccc}
\hline Año & Total & REDD & A/R & IFM \\
\hline 2009 & 098.0 & 002.8 & 004.3 & 001.3 \\
2010 & 133.0 & 018.7 & 003.0 & 003.0 \\
2011 & 095.0 & 007.3 & 007.6 & 003.0 \\
2012 & 103.0 & 006.6 & 008.8 & 003.7 \\
2013 & 076.0 & 022.6 & 002.6 & 001.2 \\
2014 & 077.0 & 025.0 & 001.6 & 000.6 \\
2015 & 084.0 & 011.1 & 003.1 & 000.7 \\
2016 & 063.0 & 009.7 & 001.3 & 001.1 \\
\hline
\end{tabular}

Fuente: Peters-Stanley, Hamilton y Yin (2012), Peters-Stanley et al. (2013), Hamrick y Goldstein (2015), Goldstein y Ruef (2016) y Hamrick y Gallant (2017b).
Un factor importante que influye sobre la oferta y demanda de los mercados voluntarios es su interacción con los mercados regulados. Como ejemplo, según Hamrick y Gallant (2017a) cuando California puso en marcha su esquema de cap and trade en 2013 varios proyectos que estaban generando créditos de compensación para el mercado voluntario, pasaron a utilizarlos para el mercado regulado. Esto explicaría la menor actividad del mercado voluntario en América del Norte en los años siguientes.

Los estándares han jugado un papel fundamental en los mercados de carbono, tanto regulados como voluntarios. En 2015, según Goldstein y Ruef (2016), 99\% de los créditos de carbono vendidos fueron verificados por estándares independientes. En los mercados voluntarios el Verified Carbon Standard (VCS) retiene la mayor cuota de mercado (en 2016: 82\% de los créditos de carbono forestal negociados; Hamrick y Gallant, 2017b) y es ampliamente utilizado en conjunto con el estándar Climate, Community and Biodiversity (CCB) que certifica los cobeneficios asociados a proyectos de carbono. A continuación el American Carbon Registry (ARC) abarcó 5\% del volumen de mercado, el Gold Standard 4\% y el Plan Vivo 2\%; siendo estos dos últimos reconocidos por asegurar cobeneficios y salvaguardas.

Los precios de venta de los créditos de carbono varían según el año, el tamaño del proyecto, su tipo, su ubicación, el tipo de mercado (primario o secundario) y si indican o no algún tipo de cobeneficio. Adicionalmente, de manera general, los proyectos grandes, que aportan mayores volúmenes al mercado suelen vender créditos a menores precios, mientras que los proyectos pequeños logran alcanzar mayores precios de venta (Peters-Stanley et al., 2013).

Los proyectos REDD+ son los que acapararon la mayor cuota de los mercados voluntarios en los años 2010, 2013, 2014 y 2016. En 2014 los proyectos REDD+ alcanzaron su record en cuanto a volumen negociado, con $25 \mathrm{Mt} \mathrm{CO}_{2} \mathrm{e}$ (Hamrick y Goldstein, 2015), mientras que en 2016 su volumen fue de $9.7 \mathrm{Mt}$ $\mathrm{CO}_{2}$ e (Hamrick y Gallant, 2017a). Estos proyectos sue- 
len generar grandes cantidades de créditos de carbono, por lo que no es extraño que sean los proyectos forestales que venden sus créditos más baratos, (entre 2007 y 2014 el precio medio de los créditos REDD+ fue de USD 5.2/t $\mathrm{CO}_{2}$ e según Hamrick y Goldstein, 2015; en 2017 los créditos REDD+ tuvieron un precio medio de USD 4.2/t $\mathrm{CO}_{2}$ e; Hamrick y Gallant, 2017a). Los cobeneficios sociales y ambientales que traen asociados estos proyectos suelen ser altamente valorados por los compradores.

En relación a REDD+ es importante diferenciar los proyectos REDD+, desarrollados por organizaciones con y sin fines de lucro, al igual que otros tipos de proyectos de carbono forestal, de los programas REDD+ que son desarrollados por los gobiernos de países o jurisdicciones (Hamrick y Gallant, 2017b). Las particularidades de REDD+ llevaron al VCS a desarrollar una metodología jurisdiccional (Jurisdictional and Nested REDD+) como un marco de contabilidad y verificación para programas REDD+ jurisdiccionales y proyectos anidados (VCS, 2018). Entre los proyectos piloto de esta iniciativa se incluyen el estado de Acre (Brasil), Costa Rica, Perú y la República Democrática del Congo.

Los proyectos $A / R$ alcanzaron sus mayores volúmenes de venta de créditos en el mercado voluntario en los años 2011 y 2012, coincidiendo con el final del primer periodo de cumplimiento del PK; posteriormente sus volúmenes han descendido, situándose en $1.3 \mathrm{Mt} \mathrm{CO}_{2} \mathrm{e}$ en 2016 (Hamrick y Gallant, 2017a), aunque el precio de venta de los créditos ha permanecido alto (precio medio 2007-2014: USD 7.7/t CO 2 e; Hamrick y Goldstein, 2015),

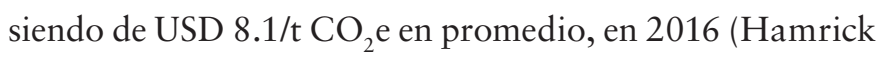
y Gallant, 2017a).

Los proyectos IFM han tenido una mayor relevancia en los mercados regulados, como el de California. En los mercados voluntarios negociaron su mayor volumen en 2012 (3.7 Mt $\mathrm{CO}_{2} \mathrm{e}$ ) y en $2016 \mathrm{el}$ volumen fue de $1.1 \mathrm{Mt}$ $\mathrm{CO}_{2} \mathrm{e}$; no obstante, los precios de los créditos asociados han logrado alcanzar precios elevados (precio medio 2007-2014: USD 8.4/t CO 2 e, Hamrick y Goldstein, 2015), situándose en promedio en 2016 en USD 9.5/t $\mathrm{CO}_{2} \mathrm{e}$.

\section{Experiencias en México}

México, como país comprometido con la lucha contra el cambio climático y miembro de la Cmnucc, cuenta con compromisos de reducción de emisiones, los cuales se contemplan en la Ley General de Cambio Climático (2012) que establece una meta aspiracional de reducir las emisiones de $\mathrm{CO}_{2}$ e al 2020 en $30 \%$ y a $50 \%$ en 2050, con respecto a las registradas en el año 2000. La ley señala que estas metas podrán alcanzarse si se establece un régimen internacional que disponga de mecanismos de apoyo financiero y tecnológico por parte de países desarrollados hacia países en desarrollo, como México. También, en 2012, el Gobierno Federal elaboró el documento "Bases para una Estrategia de Desarrollo Bajo en Emisiones en México", con el objetivo de marcar el camino para alcanzar las metas nacionales de mitigación (Semarnat, 2012). En el documento se analiza el potencial estimado de abatimiento de emisiones en México y se realiza un diagnóstico sectorial, que incluye la generación y consumo de energía, el transporte, manejo de residuos y uso de la tierra. En relación con el uso de la tierra, el potencial teórico de mitigación estimado para el 2020 en el sector forestal es de $57 \mathrm{Mt} \mathrm{CO}_{2}$ e $(20 \%$ del potencial de abatimiento nacional), basándose las oportunidades identificadas en evitar la pérdida y degradación de la cobertura forestal.

Adicionalmente, en los "Compromisos de mitigación y adaptación ante el cambio climático para el periodo 2020-2030" (Gobierno de la República, 2014), que es la contribución de México al acuerdo global sobre el clima, el país contempla la reducción de $22 \%$ de sus emisiones de GEI al 2030, lo que implica reducir $210 \mathrm{Mt}$ $\mathrm{CO}_{2}$ e. Para el sector Uscusys, las emisiones comprometidas son de -14 Mt $\mathrm{CO}_{2}$ e (absorciones) (Gobierno de la República, 2014).

A continuación, se presentan las principales experiencias desarrolladas en México en relación a la implementación de iniciativas, mecanismos y proyectos para la mitigación de emisiones relacionadas con el sector forestal y los mecanismos financieros empleados, así como los que se encuentran en desarrollo actualmente y tienen potencial para incluir al sector forestal. 


\section{Proyectos del MDL}

En México, a julio de 2017, se habían contabilizado 225 proyectos MDL, $53 \%$ de los cuales son proyectos de reducción de emisiones de metano. Destaca el hecho de que no se han registrado proyectos de tipo A/R. Según el Inecc (2016a), sin embargo, hay un proyecto $A / R$ que cuenta con carta de aprobación, que podría reducir 1,826 $\mathrm{tCO}_{2} \mathrm{e}$ anuales, pero no ha sido registrado ante la Junta Ejecutiva del MDL.

\section{NAMAs}

México ha creado un Registro Nacional de NAMAs para conocer las iniciativas que se están desarrollando en el país, centralizar la información, asistir en el registro internacional y en la canalización de posibles apoyos. Este registro es voluntario e independiente de la aprobación de registro ante la Cmnucc (Inecc, 2016b). México tiene 28 NAMAs registradas a escala nacional, de las cuales, únicamente dos están en etapa de implementación: la NAMA de vivienda nueva (financiada por la NAMA facility) y el Programa de Ahorro de Eficiencia Energética empresarial (Paeeem) Eco-Crédito. De las NAMAs registradas a escala nacional 18 están relacionadas con el uso de energía y únicamente dos se relacionan con el sector forestal: acciones de reducción de emisiones y aumento de remociones de GEI en predios agropecuarios y forestales, en etapa de diseño, promovida por Desarrollo Territorial y Servicios Ambientales S. C. y Créditos de carbono con protocolos locales, a partir de la restauración forestal y el manejo holístico agropecuario, en etapa piloto, promovida por el Grupo Ecológico Sierra Gorda I. A. P.

\section{REDD+}

México ha avanzado en la construcción del mecanismo REDD + a través del desarrollo de los cuatro elementos básicos propuestos por la Cmnucc (Acuerdos de Cancún, decisión 1/CP.16). Actualmente, existe una Estrategia REDD+ Nacional (Enaredd+) que tras un largo proceso de elaboración y consulta fue aprobada en 2017 por la Comisión Intersecretarial de Cambio Climático (CICC); se tiene una propuesta de Sistema Nacional de Monitoreo
Forestal (SNMF), un nivel de referencia de las emisiones forestales (NREF) a escala nacional y un avance en la construcción de un Sistema Nacional de Salvaguardas (SNS). La Comisión Nacional Forestal (Conafor) es la institución que ha tenido el liderazgo en la construcción de estos elementos.

El financiamiento para el proceso de preparación de REDD+ en México ha procedido principalmente del Forest Carbon Partnership Facility (FCPF) del Banco Mundial, que en 2011 aprobó el Readiness Plan Idea Note (R-PIN) presentado por México en 2008, aportando USD 3.8 millones. Además del apoyo del FCPF, México ha recibido otros apoyos en su preparación para REDD+: Fondos del Programa de Inversión Forestal del Banco Mundial (en colaboración con el BID), del Gobierno de Noruega (proyecto "Fortalecimiento del proceso de preparación para REDD+ en México y el fomento a la Cooperación Sur-Sur”, en colaboración con el PNUD), del programa ONU-REDD+, entre otros.

El proceso de preparación de México para REDD+ ha permitido trabajar en tres vías: el diseño participativo de instrumentos de planeación como la Visión de REDD+ para México y la Enaredd+, el desarrollo de sus arreglos institucionales a través de la CICC y la implementación de acciones tempranas REDD+ (Atredd+) con el objetivo de generar experiencias y lecciones aprendidas.

Estas Atredd+ fueron establecidas por la Conafor en 2011, con el fin de articular esfuerzos institucionales con base en diferentes instrumentos de política pública a escala subnacional (regional y local) para abordar las causas de la pérdida de los bosques y del carbono forestal, generando oportunidades para el desarrollo de las comunidades (Conafor, 2015a). Los estados seleccionados para implementar las Atredd+ fueron Jalisco, Campeche, Quintana Roo, Yucatán y Chiapas.

En estas Atredd+ la Conafor diseñó un modelo de intervención (Conafor, 2015a) basado en el desarrollo rural sostenible. La implementación de este modelo y del esquema de pago por resultados en áreas piloto, se está realizando a través de la Iniciativa de Reducción de Emisiones (IRE), apoyada por el FCPF. En México, la IRE 
busca proveer incentivos positivos para reducir las emisiones de GEI del sector forestal (Conafor, 2016a), al tiempo que se protegen los bosques, se conserva la biodiversidad y se mejoran los medios de vida de poblaciones indígenas y comunidades locales dependientes de los bosques.

Las actividades específicas de la IRE se plasmaron en instrumentos de planeación a mediano plazo llamados Programas de Inversión (PI). Los PI establecen las actividades a realizar en los cinco años de duración de la IRE (Tabla 7). Además, se planean actividades de segunda etapa que servirán para fortalecer y ampliar las actividades iniciales y que serán financiadas con recursos del pago por resultados de la reducción de emisiones cuantificadas y comparadas con el nivel de referencia de emisiones documentado en la IRE, el cual asciende a $24024613 \mathrm{tCO}_{2} \mathrm{e}$ con una incertidumbre de 4\% (Conafor, 2016a). El nivel de referencia abarca las emisiones por deforestación y degradación forestal, incluyendo las emisiones de $\mathrm{CH}_{4} \mathrm{y}$ $\mathrm{N}_{2} \mathrm{O}$ asociadas a la degradación por incendios forestales en ecosistemas sensibles al fuego en los cinco estados participantes en la IRE. El objetivo de reducción de emisiones de la iniciativa es de $8.6 \mathrm{MtCO}_{2}$ e en cuatro años.

La inversión total requerida para la implementación de la IRE fue estimada en MXN 7990294 768, requiriéndose el primer año MXN 1732698 271. A este respecto, la Conafor ha destinado, a través del Programa de
Apoyos para el Desarrollo Forestal Sustentable (Pronafor) al menos, MXN $26.5 \times 10^{6}$ (Tabla 8; Conafor, 2016b).

Como se observa en la tabla 8 , cincuenta y seis por ciento de las ayudas corresponden al componente $\mathrm{V}$, es decir, el programa de pagos por servicios ambientales. Por otra parte, el estado que recibió un mayor monto de apoyo fue Quintana Roo, seguido de Chiapas, Yucatán, Campeche y Jalisco.

México está aproximándose a la firma de Erpas con el FCPF. El Documento de la Iniciativa de Reducción de Emisiones (ERPD, por sus siglas en inglés) fue evaluado positivamente por el FCPF (cumplimiento de $90.3 \%$ del Marco Metodológico del Fondo de Carbono), aunque se consideró que el país todavía tiene que resolver algunos aspectos legales relacionados con los derechos del carbono (FCPF, 2016). En relación a la distribución de beneficios, se considera que México todavía no justifica suficientemente cómo los planes de distribución de beneficios cumplen con la normativa internacional. Conviene destacar que, como la deforestación y degradación de ecosistemas forestales son actividades no permitidas en la legislación nacional, se argumenta que la titularidad de las emisiones evitadas corresponde al Gobierno de México, quien recibirá el pago por resultados y establecerá los mecanismos para que los beneficios derivados de este pago se canalicen a las entidades federativas para

Tabla 7. Actividades genéricas identificas en la Iniciativa de Reducción de Emisiones.

- Mejora del sistema de producción de milpa, intensificación de la agricultura tradicional y agricultura de conservación

- Ganadería sostenible, a través de sistemas silvopastoriles intensivos y semi-intensificación de la ganadería

- Manejo forestal sostenible y de vida silvestre

- Renovación y rehabilitación de cafetales

- Desarrollo de la apicultura

- Reconversión productiva

- Proyectos productivos para aumentar el ingreso

- Fortalecimiento de la gobernanza local

- Pago por servicios ambientales

- Fortalecimiento de instrumentos regulatorios

Fuente: Conafor (2016a). 
Madera y Bosques vol. 24, núm. especial, e2401913 Invierno 2018

Tabla 8. Apoyos (MXN) de Pronafor a las Áreas de Atención REDD+ en 2017.

\begin{tabular}{ccccccc}
\hline Componente & Jalisco & Chiapas & Campeche & Q. Roo & Yucatán & Total \\
\hline I & 335000 & 1155000 & 1996749 & 700000 & 1040000 & 5226749 \\
II & & 878262 & 964290 & 1067560 & & 2910112 \\
III & S. D. & S. D. & S. D. & S. D. & S. D. & S. D. \\
IV & & 1134797 & 729777 & 1760365 & & 3624939 \\
V* & 2698981 & 3232412 & 414655 & 3699488 & 4785801 & 14831338 \\
Total & 3033981 & 6400471 & 4105471 & 7227413 & 5825801 & 26593138 \\
\hline
\end{tabular}

Componentes: I) Estudios y Proyectos; II) Gobernanza y Desarrollo de Capacidades, III) Restauración forestal y Reconversión, IV) Silvicultura, abasto y transformación, V) Servicios Ambientales; S. D.: Sin datos.

*Este dato se calculó dividiendo entre cinco los valores para cinco años de los resultados por la Conafor para el componente $V$ de las Atredd.

Fuente: Conafor (2O16b)

que se lleven a cabo las actividades de segunda etapa en las áreas de intervención, las cuales serán identificadas con los dueños y habitantes de los terrenos forestales que participaron en los esfuerzos para reducir las emisiones, a través de un proceso participativo como parte del diseño de los arreglos locales para la distribución de beneficios (Conafor, 2016a).

Adicionalmente a los esfuerzos nacionales, varias entidades federativas han estado trabajando para adecuar el mecanismo REDD+ nacional a las necesidades estatales, buscando también ampliar el marco de actuación más allá de las Atredd+ de la Conafor y recursos de otras fuentes financieras. En esta línea, varios estados cuentan con o han creado fondos estatales (i.e., el Fondo Climático de la Península de Yucatán) para financiar proyectos ambientales, que podrían utilizarse para ampliar los pagos por resultados de mecanismos REDD+ o canalizarlos hasta la escala local.

\section{Proyectos en el mercado voluntario de carbono en} México

Paralelamente a los esfuerzos llevados a cabo en el marco de la Cmnucc, en México se han diseñado e implementado varios proyectos forestales para la venta de créditos en el mercado voluntario de carbono. En la tabla 9 se presentan los proyectos validados en México por alguno de los estándares del mercado voluntario o normas nacionales.
El Proyecto Scolel Te fue el primer proyecto de carbono forestal certificado por un estándar internacional en México, en este caso el Plan Vivo, el cual asegura cobeneficios sociales y ambientales. El proyecto lleva activo desde 1997 y ha vendido 518613 certificados Plan Vivo. Este proyecto contó en sus inicios con financiamiento proveniente del Programa de Investigación Forestal del Departamento para el Desarrollo Internacional del Gobierno del Reino Unido y el apoyo de investigadores del Centro de Manejo de Carbono de Edimburgo y de El Colegio de la Frontera Sur (de Jong, Tipper y Soto-Pinto, 2004). En un inicio, la Federación Internacional del Automóvil era su principal comprador de créditos de carbono; sin embargo, en la actualidad el proyecto cuenta con compradores diversos, tanto nacionales como internacionales (Zeromission, Presidencia de la República, Climate Stewards, Huella Azul, U\&We, entre otros; [Plan Vivo, 2017]).

En la Sierra Gorda se implementó el proyecto "Captura de Carbono en Comunidades de Extrema Pobreza en la Sierra Gorda de México", que fue el primer proyecto forestal en México que consiguió la certificación por el VCS y el CCB, sin embargo, debido a problemas causados por el descortezador del pino (Dendroctonus ssp.), el proyecto no pudo continuar activo, por lo que no buscó la verificación por el VCS y la validación que recibió del CCB expiró en 2016. Actualmente, la Alianza por la Sierra Gorda, ha implementado la iniciativa Planeta Carbono 
Tabla 9. Proyectos con venta de créditos de carbono en el mercado voluntario vigentes en México.

\begin{tabular}{|c|c|c|c|c|c|c|}
\hline Proyecto & Actividades & Estándar & Localización & $\begin{array}{c}\text { Año de } \\
\text { operación }\end{array}$ & $\begin{array}{l}\text { Superficie } \\
\text { (ha) }\end{array}$ & $\begin{array}{l}\text { Créditos ven- } \\
\text { didos }\left(\mathrm{CCO}^{2} e\right)\end{array}$ \\
\hline Scolel Te & $\begin{array}{l}\text { Forestación } \\
\text { Sist. agroforestales } \\
\text { Reforestación } \\
\text { Restauración forestal } \\
\text { Deforestación evitada }\end{array}$ & Plan Vivo & Chiapas & 1997 & 8958.25 & 518613 \\
\hline Sierra Gorda & Forestación & VCS-CCB & Querétaro & 1997 & 360 & 19392 \\
\hline $\begin{array}{c}\text { Fresh Breeze } \\
\text { Afforestation Project }\end{array}$ & Forestación & VCS & $\begin{array}{l}\text { Tabasco, } \\
\text { Nayarit y } \\
\text { Chiapas }\end{array}$ & 2009 & 4269.82 & 25000 \\
\hline $\begin{array}{c}\text { Sustainable Climate- } \\
\text { Friendly Coffee }\end{array}$ & Reforestación & VCS & Oaxaca & 2014 & 292 & - \\
\hline $\begin{array}{c}\text { Captura de Carbono } \\
\text { en San Juan Lachao, } \\
\text { Oaxaca }\end{array}$ & Manejo forestal mejorado & CAR & Oaxaca & 2014 & 2388.0 & 10225 \\
\hline Carboin & $\begin{array}{l}\text { Reforestación, mantenimiento rege- } \\
\text { neración natural, enriquecimiento de } \\
\text { sistemas agroforestales, recuperación } \\
\text { de áreas degradadas, restauración de } \\
\text { áreas agrícolas degradadas }\end{array}$ & $\begin{array}{l}\text { Normas } \\
\text { ANCE }\end{array}$ & Oaxaca & 2008 & 1672.3 & 20410 \\
\hline
\end{tabular}

Neutral, que ofrece créditos de carbono para que particulares y empresas compensen su huella de carbono a un precio de MXN 250/t $\mathrm{CO}_{2}$ e (Planeta Carbono Neutral, 2017).

Los proyectos Sustainable Climate Friendly Coffee y Fresh Breeze Afforestation Project han sido validados por el VCS. El proyecto de café ha sido impulsado por organizaciones no gubernamentales (ONG's) y organizaciones de productores con el fin de enriquecer y aumentar la sombra de los cafetales con árboles nativos; todavía no ha vendido créditos, mientras que el proyecto de forestación de teca ha sido impulsado por la empresa ProTeak para el establecimiento de plantaciones comerciales de alto valor y ya ha comenzado a colocar sus primeros créditos (VCS, 2017).

En Oaxaca el proyecto "Captura de Carbono en San Juan Lachao" se construyó a partir del ejercicio piloto implementado para probar el Protocolo Forestal para
México de la Climate Action Reserve (CAR) con una inversión inicial realizada por Disney (en torno a MXN 750 000; CAR, 2016). Las actividades a desarrollar (incremento de almacenes de carbono), incluyen la producción sostenible, el aprovechamiento con mínimo impacto, la conservación y la restauración de áreas degradadas.

Por último, el proyecto Carboin es una iniciativa comunitaria que busca contribuir con la remoción y reducción de GEI mediante el manejo, conservación, mejoramiento, mantenimiento y aprovechamiento sostenible de los recursos naturales (Icico, 2017). Este proyecto es el único que no está certificado por un estándar internacional, aunque si cuenta con una certificación por parte de la Asociación de Normalización y Certificación (ANCE, A. C.), por las siguientes normas: a) NMX-SSA14064-1-IMNC-2007: Especificación con orientación, a nivel de las organizaciones, para la cuantificación e 
informe de las emisiones y remociones de GEI y b) NMXSSA-14064-3-IMNC-2007: Especificación con orientación para la validación y verificación de declaraciones sobre GEI. Adicionalmente, algunas comunidades cuentan con la certificación del Forest Stewardship Council (FSC) y de la International Organic Croop Improvement Association (OCIA). Los principales compradores de créditos de carbono de este proyecto son Chinoin, Fundación Televisa y Vivelatino (Icico, 2017).

Actualmente en México están en proceso de desarrollar nuevos proyectos de carbono forestal, que presumiblemente solicitarán la validación de algún estándar próximamente. Entre ellos, dos proyectos en Puebla que han utilizado el protocolo forestal de la CAR y están en proceso de registro y un proyecto de forestación/reforestación de manglares que ha utilizado el protocolo A/R del Gold Standard, en la costa Pacífico-Sur de México.

\section{Plataforma Mexicana del Carbono (MEXICO2)}

Esta plataforma voluntaria de comercio de carbono fue creada en 2014 con el respaldo de la Secretaría de Medio Ambiente y Recursos Naturales [Semarnat], la Embajada Británica en México, el Inecc, la Conafor, el Programa de Naciones Unidas para el Medio Ambiente (Pnuma) y SIF ICAP. MEXICO2 busca desarrollar mercados ambientales en el país desarrollando tres iniciativas (MEXICO2, 2017): a) mercados voluntarios de carbono, b) campañas socioambientales y c) ejercicio de comercio de emisiones.

En relación al mercado voluntario de carbono, la plataforma promueve la compra de créditos de carbono de proyectos de carbono ubicados en México que están certificados por algún estándar internacional (MDL, VCS, Gold Standard, Climate Action Reserve y Plan Vivo). Entre los proyectos que promueve se encuentran Scolel Te $y$ Fresh Breeze Afforestation Project (únicos relacionados con el uso de la tierra).

Las campañas socioambientales buscan invitar a empresas, instituciones e individuos a aportar donaciones a diferentes proyectos relacionados con la conservación de los recursos naturales ubicados en México, entre ellos, la conservación de la Reserva de la Biósfera Sierra Gorda.
El ejercicio de comercio de emisiones es un proyecto diseñado por la Semarnat y MÉXICO2 que consiste en hacer una simulación de cómo podría funcionar el esquema de comercio de emisiones en México. Este ejercicio pretende reproducir, mediante una herramienta digital (CarbonSIM, desarrollada por la Environmental Defense Fund), los principales componentes de un sistema de comercio de emisiones: fijación del límite de las emisiones, asignación de permisos de emisión, subastas, mercado, penalizaciones y estrategias de abatimiento (MEXICO2, 2017). En este ejercicio participan más de 100 empresas, las cuales representan $68 \%$ de las emisiones del país (MEXICO2, 2017). Toda la información generada en el proceso es ficticia y la participación de las empresas es anónima y no vinculante (no se derivan obligaciones de pago reales). El ejercicio dura nueve meses desde su inicio en tres fases de diez semanas cada una.

\section{El impuesto a los combustibles fósiles en México}

En 2014 se instauró el impuesto a la enajenación e importación de combustibles fósiles de acuerdo con su contenido de carbono en el Artículo 2 (Fracción 1, Inciso H) de la Ley del Impuesto Especial sobre Producción y Servicios (IEPS). Este impuesto, creado bajo el principio de "quien contamina paga", pretende promover la adopción de tecnologías más limpias en la producción de bienes y servicios y desincentivar las emisiones de gases de efecto invernadero que ocasionan el cambio climático (Secretaría de Hacienda y Crédito Público [SHCP], 2014).

Según el Artículo 5 de la Ley, este impuesto puede ser pagado con la entrega de bonos de carbono procedentes de proyectos de ahorro o eficiencia energética desarrollados en México. Las reglas para el pago opcional del impuesto mediante la entrega de bonos de carbono han sido realizadas por la Secretaría de Hacienda y Crédito Público y fueron publicadas en el Diario Oficial de la Federación (DOF 18/12/17). Estas reglas permiten la utilización de RCEs emitidos a partir del 01 de enero de 2014 y que correspondan al segundo periodo de compromisos del Protocolo de Kioto. Además, se establece que durante el primer año de vigencia de las reglas, el pago del 
IEPS aplicable a los combustibles fósiles mediante bonos de carbono, únicamente procederá hasta por un monto máximo de $20 \%$ de dicho gravamen que se deba pagar en las declaraciones mensuales.

\section{Mercado de comercio de emisiones}

Desde 2014 México ha realizado acciones para avanzar hacia el establecimiento de un sistema de comercio de emisiones regulado en el país.

A escala nacional se estableció el impuesto a los combustibles fósiles en 2014 y en 2017 se lanzó el ejercicio de mercado diseñado por la Semarnat y la plataforma MEXICO2. Adicionalmente, se ha reformado la Ley General de Cambio Climático para incorporar las disposiciones del Acuerdo de París y establecer el mandato para la creación de un mercado de carbono obligatorio en México.

En este sentido, la Semarnat ha anunciado que durante el primer semestre del 2018 se publicarán las reglas de mercado y la actualización de las reglas del Registro Nacional de Emisiones. El mercado operará formalmente a partir de agosto de 2018 implementándose en dos fases: Fase 1 "Piloto", con una duración de tres años (agosto 2018 - agosto 2021), posteriormente, se actualizarán las bases para arrancar formalmente el mercado de carbono en su Fase 2 "Arranque formal", coincidiendo con la entrada en vigor del Acuerdo de París.

Por otra parte, México ha buscado oportunidades para ligar el esquema de mercado que se implemente en el país con otras iniciativas desarrolladas en América. Por ello, es observador del WCI y firmó un Memorándum de Entendimiento con California en 2014, con Quebec en 2015 y con las provincias de Quebec y Ontario (Canadá), en 2016. Este último memorándum fue firmado en la segunda Cumbre de Cambio Climático de las Américas, para el desarrollo de un mercado de carbono con el objetivo de permitir que las empresas de esas provincias adquieran créditos mexicanos de reducción de GEI para satisfacer los límites de emisiones regulados por las provincias. Adicionalmente, en diciembre de 2017, México firmó la Declaración sobre el precio al carbono en las
Américas que busca promover los mercados de carbono intrarregionales y estandarizar sus sistemas de precios.

Aunque todavía se está desarrollando el esquema y no se han publicado reglas sobre el uso de compensaciones y metodologías aprobadas, se espera que, si se establece la liga con la WCI, el mercado permita el uso de créditos de compensación de proyectos forestales y relacionados con el uso de la tierra (Hamrick y Gallant, 2017b).

\section{Oportunidades de participación del sector forestal en la mitigación de emisiones de México}

En México, la meta no condicionada de mitigación señalada para el sector Uscusys en los NDC implica la reducción de $46 \mathrm{MtCO}_{2}$ e (línea de base: $32 \mathrm{MtCO}_{2}$ e; Meta: -14 $\left.\mathrm{MtCO}_{2} \mathrm{e}\right)$. Para alcanzar esta meta es necesario reducir las emisiones asociadas al cambio de uso del suelo de tierras forestales a otros usos (pastizales, agrícolas, asentamientos y otras tierras) y a los incendios y/o aumentar las absorciones asociadas al cambio de otras tierras a tierras forestales.

La participación planeada del sector forestal para lograr esta meta se centra en (Gobierno de la República, 2014): a) alcanzar en el 2030 la tasa cero de deforestación y b) mejorar el manejo forestal. La meta sobre la tasa de deforestación es compartida por los componentes de mitigación y adaptación. La Enaredd+, en sus hitos al 2030 también incluye alcanzar la tasa cero de deforestación neta y, además, reducir significativamente la tasa nacional de degradación, incrementar la superficie bajo manejo forestal sostenible y aumentar los reservorios de carbono forestal.

En cuanto a las políticas encaminadas a reducir la deforestación y degradación forestal, se implementa la IRE, la cual permitirá reducir $8.6 \mathrm{MtCO}_{2}$ e en cuatro años (Conafor, 2016a), por las actividades a realizarse en las áreas de intervención, que abarcan $21 \%$ de la superficie nacional de bosque.

Sin embargo, en el periodo 2007-2010 las emisiones por deforestación bruta en México han sido estimadas en $27.3 \mathrm{MtCO}_{2} \mathrm{e}$ año ${ }^{-1}$ y por degradación forestal en 1.8 $\mathrm{MtCO}_{2} \mathrm{e}$ año ${ }^{-1}$ (Conafor, 2015b), por lo que, todavía 
podrían reducirse $20.5 \mathrm{MtCO}_{2} \mathrm{e}$ de las emisiones asociadas a la deforestación y degradación forestal a través de actividades que incrementen el valor de los bosques en pie, como el manejo forestal. De cualquier forma, las emisiones a reducir variarán en función de la línea base considerada y de la evolución de las tasas de deforestación y degradación en los próximos años.

Para lograr una efectiva mitigación de emisiones en el sector forestal y así avanzar hacia el cumplimiento de las metas comprometidas, México cuenta con las experiencias a nivel de proyectos de carbono forestal implementadas en el país y con los aprendizajes del proceso de preparación para REDD+ y la IRE, entre otros. Además, mirando hacia el futuro, el país se encuentra desarrollando un esquema de comercio de emisiones que se pretende sea un instrumento clave para cumplir con el objetivo de reducción planteado en los NDC y, para su diseño, serán de gran valor las experiencias desarrolladas a escala internacional.

Los proyectos de carbono forestal efectuados en el país han generado experiencia y proporcionan información básica sobre la importancia del financiamiento inicial que, en la mayoría de los casos, ha sido aportado por el sector privado, los retos asociados al proceso de implementación de los proyectos, los beneficios ambientales relacionados con el carbono y más allá del carbono y los beneficios sociales y económicos para las comunidades rurales. Además, aportan datos y estimaciones sobre la magnitud de la absorción/reducción de emisiones que es posible alcanzar con distintos tipos de proyectos. En la tabla 10 se muestran los datos de captura de carbono estimados para distintas actividades asociadas a proyectos de carbono desarrollados en México. Adicionalmente, la Conafor ha estimado que la incorporación de los bosques y selvas de México al manejo forestal sostenible podría traducirse en beneficios de mitigación entre 0.52 y 1.05 $\mathrm{MtCO}_{2}$ e (Inecc-Semarnat, 2015).

El fomento del manejo forestal sostenible supone una importante área de oportunidad para México. Actualmente, la producción maderable en el país está centrada en el aprovechamiento de las especies del Género Pinus
TABLA 10. Incremento de los almacenes de carbono estimado en actividades relacionadas con el manejo forestal sostenible y el incremento de los almacenes de carbono forestales.

\begin{tabular}{|c|c|c|}
\hline $\begin{array}{c}\text { Componente } \\
\text { REDD+ }\end{array}$ & Actividad & $\begin{array}{l}\text { Incremento de alma- } \\
\text { cenes de carbono } \\
\left(\mathrm{tCO}_{2} \text { e } \mathrm{ha}^{-1} \text { año-1) }\right.\end{array}$ \\
\hline $\begin{array}{c}\text { Manejo forestal } \\
\text { sostenible }\end{array}$ & $\begin{array}{l}\text { Aprovechamiento } \\
\text { maderable de bos- } \\
\text { ques de pino-encino }\end{array}$ & $\begin{array}{l}12.4 \text { (promedio de } \\
\text { dos años) }\end{array}$ \\
\hline \multirow[t]{4}{*}{$\begin{array}{l}\text { Incremento de } \\
\text { los almacenes } \\
\text { de carbono }\end{array}$} & $\begin{array}{c}\text { Restauración sub- } \\
\text { tropical en bosques } \\
\text { degradados }\end{array}$ & 1.10 \\
\hline & $\begin{array}{c}\text { Acahual mejorado } \\
\text { subtropical (pino- } \\
\text { encino) }\end{array}$ & 1.10 \\
\hline & $\begin{array}{c}\text { Acahual mejorado } \\
\text { tropical (cedro y } \\
\text { caoba) }\end{array}$ & 3.52 \\
\hline & $\begin{array}{c}\text { Sombra y produc- } \\
\text { ción maderable en } \\
\text { cafetales }\end{array}$ & 1.43 \\
\hline
\end{tabular}

Fuente: Estimaciones realizadas a partir de la información de Plan Vivo (2017) e Icico (2017).

$\left(5.0 \times 10^{6} \mathrm{~m}^{3} \mathrm{r} ; 75.1 \%\right.$ de la producción nacional), seguido de los encinos (Quercus spp.; $0.7 \times 10^{6} \mathrm{~m}^{3} \mathrm{r} ; 11 \%$ de la producción nacional), siendo el principal producto la madera para aserrío (Semarnat, 2016). En 2016, los estados de la IRE contribuyeron, en su conjunto, con $9.5 \%$ de la producción maderable nacional, correspondiendo $5.1 \%$ al estado de Jalisco. Los estados tropicales, cuentan con menores extensiones de bosques templados, que son los preferidos para el aprovechamiento forestal en México, lo que explica su menor volumen de producción maderable. Por ello, el incrementar las áreas bajo manejo forestal sostenible en los estados de la IRE y áreas potenciales identificadas por la Conafor a escala nacional puede generar beneficios tanto en términos climáticos, como económicos y sociales. Por otra parte, el manejo forestal para productos no maderables y los proyectos relacionados con el 
incremento de los almacenes de carbono, aumentan el valor de los bosques secundarios en pie (e.g., plantación de especies forestales comerciales), lo que también puede repercutir en una menor degradación forestal y deforestación a escala local.

Los proyectos de forestación/reforestación, también pueden contribuir a disminuir las emisiones del sector Uscusys, ya que las absorciones debidas a la conversión de tierras en tierras forestales son contabilizadas en este sector (Inecc-Semarnat, 2015). En la tabla 11 se muestran las absorciones de carbono calculadas en los proyectos forestales de carbono de tipo A/R implementados en México.

Según las estimaciones de las tablas 10 y 11, el implementar actividades de manejo forestal sostenible podría aportar 1.2 $\mathrm{MtCO}_{2} \mathrm{e}$ al año, el incremento de los almacenes de carbono forestal entre $0.1 \mathrm{MtCO}_{2} \mathrm{e}-0.4 \mathrm{MtCO}_{2} \mathrm{e}$ al año y las plantaciones forestales entre $0.4 \mathrm{MtCO}_{2}$ e y 1.2 $\mathrm{MtCO}_{2}$ e al año, considerando en cada caso una superficie de 100000 ha. Para obtener mejores estimaciones sería necesario consultar fuentes adicionales de información, destacándose el estudio realizado por Casiano, Paz, Rojo, Covaleda y Aryal (2018), en este mismo número, sobre el incremento de carbono en ecosistemas forestales de México con un enfoque de cronosecuencias.

Las diferentes formas en que ha sido incluido el sector forestal en los esquemas de mercado desarrollados a escala

TABLA 11. Incremento de los almacenes de carbono estimado en actividades de forestación/reforestación de proyectos de carbono en México.

\begin{tabular}{cc}
\hline Actividad & $\begin{array}{c}\text { Incremento de almacenes } \\
\text { de carbono } \\
\left(\mathrm{tCO}_{2} \mathrm{e} \mathrm{ha}^{-1} \mathrm{año}^{-1}\right)\end{array}$ \\
\hline Plantaciones de teca & 12.3 \\
\hline Plantaciones de cedro y & 3.63 \\
caoba en sistema de taungya & 8.02 \\
\hline Plantaciones de pino & \\
\hline
\end{tabular}

Fuente: Estimaciones realizadas a partir de la información de VCS (2017) y Plan Vivo (2017). internacional y las razones que llevaron a los países a adoptar una aproximación u otra, constituyen una importante fuente de lecciones aprendidas de las que pueden aprovecharse los países que actualmente están comenzando a desarrollar sus propios esquemas y muestran que es posible ir adaptando y cambiando los enfoques en función de nuevas necesidades u oportunidades. Estos mecanismos de mercado pueden, de hecho, considerarse como instrumentos de política ambiental (Voss, 2007), que han sido impulsados a escala global principalmente desde la entrada en vigor del PK.

Desde 2005, los países con metas de reducción de emisiones implementaron mecanismos financieros basados en el mercado, al entenderse que éste era un método costo-efectivo para que las entidades reguladas alcanzaran sus metas de mitigación. Diversos países y la Unión Europea implementaron experiencias que evolucionaron con el tiempo, con el fin de adaptarse a su realidad y necesidades. En este sentido, se generaron situaciones contrastantes, como el hecho de que la Unión Europea no admite proyectos forestales en el EU-ETS, mientras que el primer sector que reguló Nueva Zelanda en su esquema de comercio de emisiones fue el sector forestal y, en otros mercados como el de California, los créditos de compensación forestales son los más utilizados. Australia, por su parte, es un buen ejemplo de adaptación de sus mecanismos financieros a las necesidades nacionales, pasando de un esquema de mercado a otro en el que, a través de un fondo, el gobierno es el único comprador de los bonos. En ese país los proyectos forestales y relacionados con el uso de la tierra también han jugado un papel primordial como fuente de créditos de compensación.

En el caso de México, la forma en que será incluido el sector forestal en el esquema de mercado todavía no está clara, aunque, dados los acuerdos firmados con los estados y provincias participantes en el WCI es posible que México se incline por utilizar el esquema adoptado en California de aceptar cierta cantidad de créditos de compensación como mecanismo de flexibilidad para que las empresas puedan cumplir con sus límites de emisiones. 
En este sentido, el Protocolo Forestal para México del CAR constituye un avance a tener en cuenta ya que se centra en el manejo forestal sostenible, el cual se incluye como estrategia clave para cumplir con las contribuciones determinadas a escala nacional y alcanzar los hitos de la Enaredd+. Además, al haber sido desarrollado en colaboración con entidades de California, tiene buenas posibilidades de ser aprobado como protocolo de compensación, en una eventual liga entre México y la WCI.

Otra línea de acción en la relación México-California, ha sido el debate sobre la inclusión de créditos REDD+ procedentes de esquemas jurisdiccionales, particularmente del estado de Chiapas. Sin embargo, para implementar esquemas de este tipo hay que tener en cuenta que el gobierno federal de México podría imponer restricciones a los gobiernos subnacionales para acceder a beneficios de reducción de emisiones de forma independiente, ya que no podrían ser tomados en cuenta en la contabilidad nacional. En relación a esto, una particularidad de los pagos basados en resultados para $\mathrm{REDD}+$ es que los compradores no han reclamado las reducciones de emisiones adquiridas para su propia contabilidad (Hamrick y Gallant, 2017b), lo cual, en caso de cambiar en el futuro podría poner en riesgo el cumplimiento de las metas nacionales de los países generadores de créditos REDD+.

Actualmente, el único esquema de compensación de emisiones activo en México es el asociado al IEPS, que con las reglas actuales sólo permite la utilización de proyectos MDL, lo cual, en este momento, limita enormemente la participación del sector forestal, debido a que, los únicos proyectos relacionados con este sector bajo el MDL son los proyectos A/R y actualmente México no tiene registrado ningún proyecto de este tipo. El considerar únicamente certificados avalados por el MDL, además de limitar los tipos de proyectos forestales a participar, crea pocos incentivos para los implementadores de proyectos por los bajos precios de los RCEs en los últimos años (precio medio en 2016: USD 1.6/ $\mathrm{tCO}_{2} \mathrm{e}$; Hamrick y Gallant, 2017a) y la incertidumbre sobre el futuro del mecanismo, en el marco del Acuerdo de París.
Con una visión más amplia, un esquema de compensación asociado al sistema de comercio de emisiones de México podría aceptar créditos de proyectos de manejo forestal e incremento de los almacenes de carbono, así como créditos REDD+, en consonancia con las metas de mitigación nacionales. Una opción es considerar un esquema que admita créditos de programas jurisdiccionales, a escala de entidad federativa, que incluya proyectos anidados, en línea con lo propuesto por el VCS (2018), de tal forma que se eviten dobles contabilidades. Dentro de este esquema, la admisión de créditos procedentes de NAMAs acreditadas que se desarrollen en el sector forestal podría valorarse, permitiendo así la incorporación al esquema de mercado de iniciativas en desarrollo.

En este contexto, los esfuerzos de la IRE podrían expandirse a todo el territorio de los estados en los que opera, en los que, en conjunto, todavía quedan casi 11000000 ha de bosque fuera de las áreas de intervención. Por otra parte, además de los estados de la IRE, otras entidades se han mostrado muy activas para implementar programas REDD+ estatales, tales como Chihuahua, Oaxaca, Tabasco o el Estado de México.

Por último, conviene tener en cuenta que los mecanismos basados en pagos por resultados que se implementen, deberán considerar esquemas de distribución de beneficios que sean transparentes, equitativos y sencillos. Las ventajas y desventajas de las distintas opciones han sido discutidas en Skutsch et al. (2014) y la importancia de considerar esquemas que no excluyan a grupos vulnerables ha sido tratada en Skutsch, Balderas y Carrillo (2017).

\section{CONCLUSIONES}

Para conseguir la reducción de emisiones en el sector Uscusys, México debe lograr una efectiva coordinación entre políticas forestales y de cambio climático y un óptimo aprovechamiento de los mecanismos financieros que se están gestando, destacándose la experiencia de la IRE y el desarrollo del esquema regulado de comercio de emisiones. En este sentido, la aceptación de créditos de compensación procedentes de distintos tipos de proyectos 
forestales, en una visión más amplia a la contemplada en las reglas para el pago opcional del IEPS a través de bonos de carbono, podría impulsar las acciones de mitigación de emisiones en el sector forestal, a la vez que se apoya a otros sectores para que alcancen sus metas.

Adicionalmente, la implementación de proyectos de carbono forestal (REDD+, manejo forestal sostenible, incremento de los almacenes de carbono) trae aparejados diferentes cobeneficios, ya que contribuye a mejorar el nivel de vida de las poblaciones locales al disponer de nuevas fuentes de ingresos, disminuir las tasas de deforestación y degradación a escala local, al darle valor al bosque en pie y contribuir a la conservación de la biodiversidad y servicios ecosistémicos.

\section{REFERENCIAS}

Agarwal, S., Bruer, V., Cameron, L. Davis, S., Escalante D., ..., \& Wienges, S. (2013). Annual Status Report on Nationally Appropriate Mitigation Actions (NAMAs). Recuperado de http://mitigationpartnership.net/sites/default/ files/mitigation_momentum_annual_status_ report_27-02-2013.pdf.

Air Resource Board [ARB]. (2017). Compliance Offset Program. Recuperado de https://www.arb.ca.gov/cc/capandtrade/offsets/offsets.htm.

Amazon Fund. (2017). Donations. Recuperado de http://www. amazonfund.gov.br/FundoAmazonia/fam/site_en/ Esquerdo/doacoes/.

Australian Government. (2017a). Carbon Farming Initiative. Recuperado de http://www.environment.gov.au/climatechange/emissions-reduction-fund/cfi/about.

Australian Government. (2017b). About the Emissions Redution Fund. Recuperado de http://www.environment.gov. $\mathrm{au} /$ climate-change/emissions-reduction-fund/about.

Carlino, H., Netto, H., Cabrera, M. M. \& Serra, L. (2017). El papel central del financiamiento en el Acuerdo de París y las oportunidades para los bancos nacionales de desarrollo. Documento para la discusión IDB-DP-497. Banco Interamericano de Desarrollo.

Casiano, M., Paz, F., Rojo, M., Covaleda, S., \& Aryal, D. R. (2018). El carbono de la biomasa aérea medido en crono- secuencias: primera estimación en México. Madera y Bosques, en prensa.

Center for Climate and Energy Solutions (2011). Australia's Carbon Pricing Mechanism. Recuperado de https://www. c2es.org/publications/australia-carbon-pricing-mechanism.

Climate Action Reserve [CAR] (2016). Projects. Recuperado de http://www.climateactionreserve.org/how/projects/.

Climate Action Reserve [CAR] (2017). Compliance Offset Projects. Recuperado de http://www.climateactionreserve. org/how/california-compliance-projects/compliance-offset-projects/.

Convención Marco de Naciones Unidas sobre el Cambio Climático [Cmnucc] (2007). Decisión 1/CP.13, Plan de Acción de Bali. FCCC/CP/2007/6/Add.1UNFCCC.

Convención Marco de Naciones Unidas sobre el Cambio Climático [Cmnucc].(2012). Enmienda de Doha al Protocolo de Kioto. Recuperado de http://unfccc.int/kyoto_protocol/ doha_amendment/items/7362.php.

Convención Marco de Naciones Unidas sobre el Cambio Climático [Cmnucc].(2013). Informe anual de la Junta Ejecutiva del mecanismo para un desarrollo limpio a la Conferencia de las Partes en calidad de reunión de las Partes en el Protocolo de Kyoto. Recuperado de https:// cdm.unfccc.int/Reference/EB_CMP_rep/index.html.

Convención Marco de Naciones Unidas sobre el Cambio Climático [Cmnucc] (2014a). La convención del cambio climático. Recuperado de http://unfccc.int/portal_espanol/ informacion_basica/la_convencion/items/6196.php.

Convención Marco de Naciones Unidas sobre el Cambio Climático [Cmnucc] (2014b). Joint Implementation. Recuperado de http://unfccc.int/kyoto_protocol/mechanisms/ joint_implementation/items/1674.php.

Convención Marco de Naciones Unidas sobre el Cambio Climático [Cmnucc] (2014c). Clean Development Mechanism. Recuperado de http://unfccc.int/kyoto_protocol/mechanisms/clean_development_mechanism/items/2718.php.

Convención Marco de Naciones Unidas sobre el Cambio Climático [Cmnucc] (2015a). The Kioto Protocol. 10 ${ }^{\text {th }}$ Anniversary, Timely Reminder Climate Agreements Work. Recuperado de http://newsroom.unfccc.int/unfccc-news- 
room/kyoto-protocol-10th-anniversary-timely-reminderclimate-agreements-work/\#downloads.

Convención Marco de Naciones Unidas sobre el Cambio Climático [Cmnucc] (2015b). The Paris Agreement. Recuperado de http://unfccc.int/paris_agreement/items/9485.php.

Convención Marco de Naciones Unidas sobre el Cambio Climático [Cmnucc] (2016a). Informe anual de la Junta Ejecutiva del mecanismo para un desarrollo limpio a la Conferencia de las Partes en calidad de reunión de las Partes en el Protocolo de Kyoto. Recuperado de https:// cdm.unfccc.int/Reference/EB_CMP_rep/index.html.

Convención Marco de Naciones Unidas sobre el Cambio Climático [Cmnucc] (2016b). Key decisions relevant for reducing emissions from deforestation and forest degradation in developing countries (REDD+). Decision booklet REDD+. Recuperado de http://unfccc.int/land_use_and_ climate_change/lulucf/items/6917.php.

Convención Marco de Naciones Unidas sobre el Cambio Climático [Cmnucc] (2017). Unfccc Overview. Recuperado de http://unfccc.int/focus/overview/items/7879.php.

Comisión Nacional Forestal [Conafor] (2015a). Modelo de intervención en las Áreas de Acción Temprana REDD+. Conafor. Zapopan, Jalisco, México.

Comisión Nacional Forestal [Conafor] (2015b). Mexico's Forest Reference Emission Level Proposal. Recuperado de http://redd.unfccc.int/files/frel_mexico_modified.pdf.

Comisión Nacional Forestal [Conafor] (2016a). Documento de la Iniciativa de Reducción de Emisiones. FCPF. Recuperado de http://www.gob.mx/cms/uploads/attachment/ file/84499/06_Iniciativa_de_Reduccion_de_Emisiones. pdf.

Comisión Nacional Forestal [Conafor] (2016b). Programa Nacional Forestal [Pronafor]. Recuperado de http:// www.conafor.gob.mx/apoyos/index.php/inicio/app_apoyos\#/detalle/2017/68.

De Jong, B. H., Tipper, R., \& Soto-Pinto, L. (2004). Proyecto Scolel Té: la participación de comunidades rurales en el mercado internacional de venta de carbono. En J. Martínez, \& A. Fernández. Cambio Climático: Una visión desde México. D. F., México: Secretaría de Medio Ambiente-Instituto Nacional de Ecología.
Díaz, D., Hamilton, K., \& Johson, E. (2011). State of the Forest Carbon Markets 2011: From Canopy to Currency. Recuperado de http://www.forest-trends.org/publication_ details.php? publicationID=2963.

Ecofys (2017). NAMA Database pipeline: April 2017. Recuperado de http://www.nama-database.org/nama-db-pipeline. $x \operatorname{lm}$.

Eguren, L. (2004). El mercado de carbono en América Latina y el Caribe: balance y perspectivas. CEPAL- Serie Medio Ambiente y Desarrollo. Santiago de Chile, Chile: Naciones Unidas.

Eliasch, J. (2008). Eliasch Review. Climate Change: Financing Global Forest. Recuperado de https://www.gov.uk/government/organisations/eliasch-review.

European Comission (2017a). Régimen de comercio de derechos de emisión de la UE (RCDE UE). Recuperado de https://ec.europa.eu/clima/policies/ets_es.

European Comission (2017b). Land use and forestry proposal for 2021-2030. Recuperado de https://ec.europa.eu/ clima/policies/forests/lulucf_en\#tab-0-0.

Forest Carbon Partnership Facility [FCPF] (2016). Technical Assessment of Final ER-PD Mexico. Recuperado de https://www.forestcarbonpartnership.org/mexico.

Forest Carbon Partnership Facility [FCPF] (2017). REDD+ Countries. Recuperado de https://www.forestcarbonpartnership.org/redd-countries-1.

Gobierno de la República (2014). Compromisos de mitigación y adaptación ante el cambio climático para el periodo 2020-2030. Recuperado de http://www.inecc.gob.mx/ descargas/adaptacion/2015_indc_esp.pdf.

Goldstein, A., \& González, G. (2014). Turning over a New Leaf. State of the Forest Carbon Markets 2014. Recuperado de http://www.forest-trends.org/fcm2014.php.

Goldstein, A., \& Neyland E. (2015). Coverging at the Crossroads: State of Forest Carbon Finance 2015. Recuperado de http://forest-trends.org/releases/p/sofcf2015.

Goldstein, A., \& Ruef, F. (2016). View from the Understory. State of the Forest Carbon Markets 2016. Recuperado de http://forest-trends.org/releases/p/sofcf2016.

Green Climate Fund [GCF] (2017). Pilot Programme for REDD+Results-based Payments. GCF/B.17/13. 26 June 
2017. Meeting of the Board. 5-6 July 2017. Songdo, Incheon, Republic of Korea.

Guyana REDD+ Investment Fund [GRIF] (2015). Guyana REDD+ Investment Fund Trust Fund. Financial Report prepared by the trustee as of December 31, 2015. Recuperado de http://www.guyanareddfund.org/index.php/trustee.

Hamilton, K., Sjardin, M., Marcello, T., \& Gordon, X. (2008). Forging a Frontier: State of the Voluntary Carbon Markets 2008. Recuperado de http://www.ecosystemmarketp l a c e.c o m / p u b l i c a t i o n s / forging-a-new-frontier-state-of-the-voluntary-carbonmarkets-2008/.

Hamilton, K., Sjardin, M., Peters-Stanley, M., \& Marcello, T. (2010). Building bridges: State of the Voluntary Carbon Markets 2010. Recuperado de http://www.forest-trends. org/publication_details.php?publicationID=2433.

Hamrick, K. (2015). New Zealand seeks to reform a floundering Emissions Trading Program. Recuperado de http:// www.ecosystemmarketplace.com/articles/new-zealandseeks-to-reform-a-floundering-emissions-tradingrogram/.

Hamrick, K., \& Goldstein, A. (2015). Ahead of the Curve: State of the Voluntary Carbon Markets 2015. Recuperado de http://forest-trends.org/releases/p/ahead_of_the_curve_ state_of_the_voluntary_carbon_markets_2015.

Hamrick, K., \& Gallant, M. (2017a). Unlocking Potential. State of the Voluntary Carbon Markets 2017. Recuperado de http://forest-trends.org/releases/p/sovcm2017,

Hamrick, K., \& Gallant, M. (2017b). Fertile Ground. State of the Forest Carbon Finance 2017. Recuperado de http:// forest-trends.org/releases/p/sofcf2017.

Integradora de Comunidades Indígenas y Campesinas de Oaxaca, A. C. [Icico] (2017). Proyecto Carboin. Recuperado de http://www.icico.org.mx/carboin.html.

Independent Pricing and Regulatory Tribunal [IPART] (2012). Compliance and Operation of the NSW Greenhouse Gas Reduction Scheme during 2012. Report to Minister. Recuperado de https://www.ipart.nsw.gov.au/Home/ Industries/Greenhouse-Gas-Reduction-Scheme/Compliance-and-Operation-of-the-NSW-Greenhouse-Gas-
Reduction-Scheme-during-2012-Report-to-MinisterDecember-2012.

Instituto Nacional de Ecología y Cambio Climático [Inecc] (2016a). Mecanismo de desarrollo limpio [MDL]. Recuperado de https://www.gob.mx/inecc/acciones-y-programas/mecanismo-de-desarrollo-limpio-mdl.

Instituto Nacional de Ecología y Cambio Climático [Inecc] (2016b). Acciones nacionalmente apropiadas de mitigación [NAMAs]. Recuperado de https://www.gob.mx/ inecc/acciones-y-programas/acciones-nacionalmenteapropiadas-de-mitigacion-namas.

Instituto Nacional de Ecología [INE] y Secretaría de Medio Ambiente y Recursos Naturales [Semarnat] (2006). Tercera comunicación nacional ante la Convención Marco de las Naciones Unidas sobre el Cambio Climático. México, D. F.: INE/Semarnat.

Instituto Nacional de Ecología [INE] y Secretaría de Medio Ambiente y Recursos Naturales [Semarnat] (2009). Cuarta comunicación nacional ante la Convención Marco de las Naciones Unidas sobre el Cambio Climático. México, D. F.: INE/Semarnat.

Instituto Nacional de Ecología y Cambio Climático [Inecc] y Secretaría de Medio Ambiente y Recursos Naturales [Semarnat] (2012). Quinta comunicación nacional ante la Convención Marco de las Naciones Unidas sobre el Cambio Climático. México, D. F.: INE/Semarnat.

Instituto Nacional de Ecología y Cambio Climático [Inecc] y Secretaría de Medio Ambiente y Recursos Naturales [Semarnat] (2015). Primer informe bienal de actualización ante la Convención Marco de las Naciones Unidas sobre el Cambio Climático. México, D. F.: INE/Semarnat. Intergovernmental Panel on Climate Change [IPCC] (2014). Technical Summary. In O. Edenhofer, R. PichsMadruga, Y. Sokona, E. Farahani, S. Kadner, K. Seyboth, A. Adler, I. Baum, S. Brunner, P. Eickemeier, B. Kriemann, J. Savolainen, S. Schlömer, C. von Stechow, T. Zwickel, \& J. C. Minx (eds.), Climate Change 2014: Mitigation of Climate Change. Contribution of Working Group III to the Fifth Assessment Report of the Intergovernmental Panel on Climate Change. United Kingdom and New York, NY, USA: Cambridge University Press, Cambridge. 
Low Emission Capacity Building Programme [LECBP] (2017). Low emission development. Recuperado de http://www. lowemissiondevelopment.org/lecbp/about-us.

Markit. (2017). Registry-Public View. Recuperado de https:// mer.markit.com/br-reg/public/index.jsp?entity=project \&sort=project_name\&dir=ASC\&start=0\&entity_ domain=Markit,GoldStandard.

MEXICO2. (2017). Medioambiente. Recuperado de http:// www.mexico2.com.mx/medio-ambiente.php?id=5.

Nabuurs, G. J., Delacote, P., Ellison, D., Hanewinkel, M., Lindner, M., Nesbit, M., Ollikainen, M., \& Savaresi, A. (2015). A new role for forest and the forest sector in the EU post-2020 climate targets. From Science to Policy 2. European Forest Institute. Recuperado de www.efi.int/ files/attachments/publications/efi_fstp_2_2015.pdf.

NAMA facility (2017). Projects. Recuperado de http://www. nama-facility.org/projects/?no_cache $=1$.

New Zealand Ministry for the Environment. (2017). New Zealand Emissions Trading Scheme Review: Improving the ETS framework. Recuperado de http://www.mfe.govt.nz/ node/23492.

Peters-Stanley, M., Hamilton, K., \& Yin, D. (2012). Leveraging the Landscape. State of the Forest Carbon Markets 2012. Recuperado de www.forest-trends.org/documents/files/ doc_3242.pdf.

Peters-Stanley, M., \& Yin, D. (2013). Maneuvering the Mosaic: State of the Voluntary Carbon Markets 2013. Recuperado de http://www.forest-trends.org/vcm2013.php.

Peters-Stanley, M., González, G., \& Yin, D. (2013). Covering New Ground: State of the Forest Carbon Markets 2013. http://www.forest-trends.org/documents/files/SOFCMfull-report.pdf.

Planeta Carbono Neutral. (2017). Compensa tu huella. Recuperado de http://planetacarbononeutral.org/checkout_ $\mathrm{es} / \mathrm{?}=1$.

Plan Vivo. (2017). Scolel Te, México. Recuperado de http:// www.planvivo.org/project-network/scolelte-mexico/.

Puigdueta, I., \& Sanz, A. (2017). Doce años del Protocolo de Kioto. Recuperado de http://www.upm.es/UPM/SalaPrensa/Noticias? id=cdee4f93bf54a510VgnVCM1000000 9c7648a____\&fmt=detail\&prefmt=articulo.
Saynes, V., Etchevers, J. D., Paz, F. \& Alvarado, L. O. (2016). Emisiones de gases de efecto invernadero en sistemas agrícolas de México. Terra Latinoamericana, 34(1), 83-96.

Seeberg-Elverfeldt, C. (2010). Las posibilidades de financiación del carbono para la agricultura, la actividad forestal y otros proyectos de uso de la tierra en el contexto del pequeño agricultor. Departamento de Gestión de Recursos Naturales y Medio Ambiente Roma: FAO.

Secretaría de Hacienda y Crédito Público [SHCP] (2014). Impuesto a los combustibles fósiles. Recuperado de http://www.sat.gob.mx/fichas_tematicas/reforma_fiscal/ Paginas/combustibles_fosiles_2014.aspx.

Secretaría de Medio Ambiente y Recursos Naturales [Semarnat] (2012). Bases para una estrategia de desarrollo bajo en emisiones. México D. F.,: Inecc-Semarnat.

Secretaría de Medio Ambiente y Recursos Naturales [Semarnat] (2016). Anuario estadístico de la producción forestal 2016. México D. F.,: Semarnat.

Secretaría de Medio Ambiente y Aprovechamiento Sustentables de Campeche. (SemarnatCam) (2015). Programa Estatal de Acción ante el Cambio Climático. Recuperado de www.semarnatcam.campeche.gob. $\mathrm{mx} / \mathrm{wp}$-content/.../ PECC-Campeche_20150310.pdf

Secretaría de Medio Ambiente, Vivienda e Historia Natural de Chiapas [Semavi] (2011). Programa de Acción Ante el Cambio Climático del Estado de Chiapas. Recuperado de http://www.conservation.org/global/mexico/iniciativas/ Pages/paccch.aspx.

Secretaría de Recursos Naturales y Protección Ambiental del Estado de Tabasco [Sernapam](2011). Programa de acción ante el cambio climático del estado de Tabasco. Recuperado de https://tabasco.gob.mx/sites/.../programa_estatal_accion_cambio_climatico.pdf.

Stern, N. (2007). Stern Review: La economía del cambio climático. HM Treasury. Cambridge, UK.: Cambridge University Press.

Skutsch, M., Turnhout, E., Vijge, M. J., Herold, M., Wits, T., den Besten, J. W. \& Balderas, A. (2014). Options for a National Framework for Benefit Distribution and Their Relation to Community-Based and National REDD+ Monitoring. Forest, 5, 1596-1617. 
Skutsch, M., Balderas, A., \& Carrillo, J. C. (2017). Policy for pro-poor distribution of REDD+ benefits in Mexico: How the legal and technical challenges are being addressed. Forest Policy and Economics, 75, 58-66.

UNEP DTU Partnership (2017a). CDM/JI pipeline overview page. Recuperado de http://www.cdmpipeline.org/overview.htm.

UNEP DTU Partnership. (2017b). CDM projects by host region. Recuperado de http://www.cdmpipeline.org/cdm-projects-region.htm.

Verified Carbon Standard [VCS] (2017). VCS Project Database. Recuperado de http://www.vcsprojectdatabase.org/\#/vcs.

Verified Carbon Standard [VCS] (2018). Jurisdictional and Nested REDD+ (JNR). Recuperado de http://www.v-c-s.org/ project/jurisdictional-and-nested-redd-framework/.

Voss, J. P. (2007). Innovation processes in governance: the development of 'emissions trading' as a new policy instrument. Science and Public Policy, 34, 329-343.
World Bank. (2016). State and trends of carbon pricing. Washington, D. C., USA: World Bank Group. Recuperado de http://documents.worldbank.org/curated/en/5988114 76464765822/State-and-trends-of-carbon-pricing.

Manuscrito recibido el 21 de diciembre de 2017

Aceptado el 30 de junio de 2018

Publicado el 19 de diciembre de 2018

Este documento se debe citar como:

Ranero, A., \& Covaleda, S. (2018). El financiamiento de los proyectos de carbono forestal: Experiencias existentes y oportunidades en México. Madera y Bosques, 24(Núm. esp.), e2401913. doi: 10.21829/ $\mathrm{myb} / 2018.2401913$

Madera y Bosques por Instituto de Ecología A C se distribuye bajo una Licencia Creative Commons Atribución-No Comercial-Compartir Igual 4.0 Internacional. 